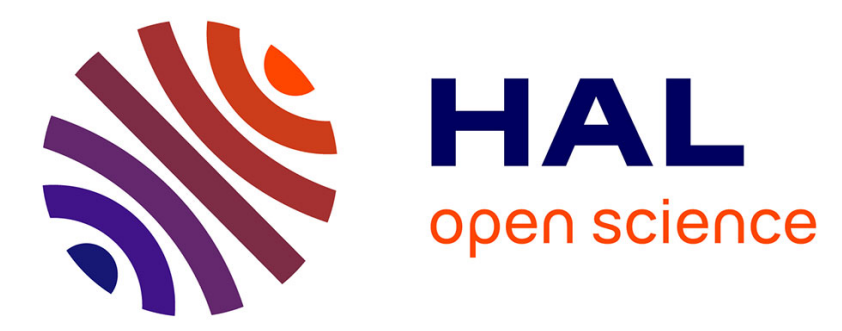

\title{
A New Approach in Nonparametric Estimation of Returns in Mean-DownSide Risk Portfolio frontier
}

Hanene Ben Salah, Ali Gannoun, Mathieu Ribatet

\section{To cite this version:}

Hanene Ben Salah, Ali Gannoun, Mathieu Ribatet. A New Approach in Nonparametric Estimation of Returns in Mean-DownSide Risk Portfolio frontier. International Journal of Portfolio Analysis and Management, 2018, 2 (2), pp.162-197. 10.1504/IJPAM.2018.092642 · hal-01299561

\section{HAL Id: hal-01299561 \\ https://hal.science/hal-01299561}

Submitted on 7 Apr 2016

HAL is a multi-disciplinary open access archive for the deposit and dissemination of scientific research documents, whether they are published or not. The documents may come from teaching and research institutions in France or abroad, or from public or private research centers.
L'archive ouverte pluridisciplinaire HAL, est destinée au dépôt et à la diffusion de documents scientifiques de niveau recherche, publiés ou non, émanant des établissements d'enseignement et de recherche français ou étrangers, des laboratoires publics ou privés. 


\title{
A New Approach in Nonparametric Estimation of Returns in Mean-DownSide Risk Portfolio frontier
}

\author{
Hanene Ben Salah ${ }^{1,2,3}$ \\ Ali Gannoun ${ }^{3} \quad$ Christian de Peretti ${ }^{2}$ \\ Mathieu Ribatet ${ }^{3}$ \\ Abdelwahed Trabelsi ${ }^{1}$
}

June 29, 2015

\author{
${ }^{1}$ BESTMOD Laboratory, ISG 41 Rue de la Liberté, Cité Bouchoucha 2000 Le Bardo,Tunisie, \\ bensalah_hanen@yahoo.fr, abdel.trabelsi@gmail.com \\ ${ }^{2}$ Institut de Science Financière et d'Assurance 69366 Lyon cedex 07, Christian.De-Peretti@ec-lyon.fr \\ ${ }^{3}$ I3M 34095 Montpellier cedex 05, ali.gannoun@um2.fr, mathieu.ribatet@um2.fr
}

\begin{abstract}
The DownSide Risk (DSR) model for portfolio optimization allows to overcome the drawbacks of the classical MeanVariance model concerning the asymmetry of returns and the risk perception of investors. This optimization model deals with a positive definite matrix that is endogenous with respect to the portfolio weights and hence yields to a non standard optimization problem. To bypass this hurdle, Athayde (2001) developed a new recursive minimization procedure that ensures the convergence to the solution. However, when a finite number of observations is available, the portfolio frontier usually exhibits some inflexion points which make this curve not very smooth. In order to overcome these points, Athayde (2003) proposed a mean kernel estimation of returns to get a smoother portfolio frontier. This technique provides an effect similar to the case in which an infinite number of observations is available. In spite of the originality of this approach, the proposed algorithm was not neatly written. Moreover, no application was presented in his paper. Ben Salah et al (2015), taking advantage on the the robustness of the median, replaced the mean estimator in Athayde's model by a nonparametric median estimator of the returns, and gave a tidily and comprehensive version of the former algorithm (of Athayde (2001, 2003)). In all the previous cases, the problem is computationally complex since at each iteration, the returns (for each asset and for the portfolio) need to be reestimated. Due to the changes in the kernel weights for every time, the portfolio is altered. In this paper, a new method to reduce the number of iterations is proposed. Its principle is to start by estimating non parametrically all the returns for each asset; then, the returns of a given portfolio will be derived from the previous estimated assets returns. Using the DSR criterion and Athayde's algorithm, a smoother portfolio frontier is obtained when short selling is or is not allowed. The proposed approach is applied on the French and Brazilian stock markets.
\end{abstract}

Keywords: DownSide Risk, Kernel Method, Nonparametric Mean Estimation, Nonparametric Median Estimation, Semivariance.

\section{Introduction}

One of the greatest contributions to the financial theory is the derivation of a formal risk-return framework by Harry Markowitz, which laid to the foundations of what we know as modern portfolio theory. Markowitz (1952) pioneered the issue of portfolio optimization with a seminal article, which was later expanded into a seminal book (Markowitz, 1959). By quantifying investment risk in the form of the variance or standard deviation of returns, Markowitz gave investors a mathematical approach to assets selection and portfolio management. Markowitz used mean return's, variances and covariances to derive an efficient frontier where each portfolio maximizes the expected return for a given variance (or minimizes variance for a given expected return). This approach is widely referred to as the MeanVariance (M-V) criterion. While modern portfolio theory of Markowitz has revolutionized the investment world, it has also received substantial criticisms. 
The main criticism concerns the use of the variance as a measure of risk since it gives the same importance and the same weights to gains and losses and supposes that returns are normally (or at least symetrically) distributed. An alternative, also suggested by Markowitz (1955), is the Semivariance which takes into consideration the asymmetry and the risk perception of investors.

In fact, normality on assets returns have been widely rejected in practice, see for example Eftekhari and Satchell (1996). This fact justifies the use of Semivariance when the presence of skewness or any other measure of asymmetry are observed.

The Semivariance is often considered as a more plausible risk measure than the variance. However academics and practitioners are still using the Mean-Variance approach for portfolio optimization as it is easier to compute, have well-known closed-form solutions and deals with a symmetric and exogenous covariance matrix, whereas MeanSemivariance optimal portfolios cannot be easily determined. This follows from the fact that, the Semicovariance matrix is endogenous and not symmetric (see Estrada 2004, 2008).

Athayde (2001) has developed an algorithm to construct a Mean-DSR portfolio frontier. This curve is continuous and convex. But nevertheless, this convexity presents many kinks due to the fact that the asset returns are not identically distributed. Clearly, the frontier is made on segments of parabolas (piecewise of quadratic functions), each one becoming steeper and steeper as we move to toward the extremes, in either direction. They are connected to each other producing the successive kinks. The more observations we have, the more parabolas will appear and the smaller the segment of each will become. Otherwise, the number of kinks in the convexity will increase with the number of observations, getting closer and closer to each other, until when we reach the asymptotic limit, they will not be qualified as kinks any more, and the whole portfolio frontier will have a smooth shape. In practice they are only a finite number of observations. Thus, when computing a DSR portfolio frontier we will always be facing these kinks on the convexity, which would only disappear if an infinite number of observations is available. In this limit case, each of the parabolas will degenerate to a single point, creating a continuous smooth changing in the convexity of the curve. However, it is not realistic to have an infinite number of observations.

In order to overcome that, Athayde (2003) used nonparametric techniques to estimate smooth continuous distribution of the portfolio in question. He proposed to replace all the returns by their mean kernel estimators counterpart, and from these estimates, he optimized their DSR, constructing with this, a new portfolio frontier. Ben Salah et al (2015) rewrote and implemented meticulously Athayde's algorithm. Then, they proposed a new approach based on median kernel estimation of the returns, and compared their method to Athayde's one. One can see that results obtained by the median estimation method are better than those obtained by the mean estimation one: a smaller DSR and a smoother frontier. Nevertheless, the computing step and the convergence of the algorithm take a long time due to the construction of the estimators: the asset estimation returns are derived from the estimation of the portfolio returns and they change at each computing step. Some convergences are obtained by imposing arbitrarily a shut-off parameter.

In this paper, alternative nonparametric methods to construct portfolio frontiers are proposed. Contrary to Athayde and Ben Salah et al' s procedure, the idea is to start by estimating all the returns of each asset using kernel mean or median estimates. The portfolio returns estimates are then obtained as a linear combination of the different assets returns estimates and the convergence is ensured without the use of any shut-off criterion. Moreover, the optimization problems are resolved using long only and long-short strategies.

This article is organized into five sections. Section 2 presents the underlying concepts of the DownSide Risk and gives an overview on Athayde optimization algorithm to select an optimal portfolio. Section 3 is devoted to a new nonparametric approach which is more efficient than the previous methods from a computing point of view. Application on data are presented in Section 4 . Conclusion and further developments are postponed to Section 5

\section{Classical Mean-DownSide Risk Model}

\subsection{Formal Framework}

Markowitz (1952) has introduced the M-V model in portfolio optimization. The objective of M-V model is to minimize the portfolio variance at a given level mean return. Covariance matrix of assets returns need to be calculated to compute portfolio variance. Variance measures the deviation above and below the mean return. Variance is not an appropriate risk measure because it does not only penalises the DownSide Deviation but also the upside one. 
DownSide Risk (DSR) is an appropriate investment risk measure because investors are more concerned about losses below a target returns. To overcome the weaknesses of M-V model, Markowitz (1959) proposed and studied the DSR alternative model where the risk is measured with the Semivariance rather than the variance, i.e.,

$$
\text { SemiVar }=\frac{1}{T} \sum_{t=1}^{T} \min \left(r_{p t}-\mu, 0\right)^{2}
$$

where $r_{p t}, t=1, \ldots, T$, are $T$ observations of the portfolio return $r_{p}$ and $\mu$ the expected return estimator (arithmetic mean of the observations).

More generally, for a given benchmark value $B$, the DownSide Risk is a generalization of the Semivariance, i.e,

$$
D S R=\frac{1}{T} \sum_{t=1}^{T} \min \left(r_{p t}-B, 0\right)^{2}
$$

Optimizing asset allocation consists in attributing weights to each asset of a given portfolio subject to constraints of an investor's capital resources to yield the most favourable risk-return trade-off.

Let $\omega=\left(\omega_{1}, \ldots, \omega_{n}\right)$ the portfolio vector weights, namely, $\omega_{i}(\in \mathbb{R})$ is the fraction of the investor's portfolio invested in the $i$-th asset, and $r_{p t}=r_{p t}(w)=\omega_{1} r_{1 t}+\cdots+\omega_{n} r_{n t}$ where $r_{i t}$ is the return of the asset $i$ at time $t$. The expected return estimator of asset $i$ is denoted by $\mu_{i}$. Using Downside Risk criterion, the optimization model is

$$
\underset{\omega}{\arg \min } \frac{1}{T} \sum_{t=1}^{T} \min \left(r_{p t}-B, 0\right)^{2} \quad \text { subject to } \sum_{i=1}^{n} \omega_{i} \mu_{i}=E_{*} \text { and } \sum_{i=1}^{n} \omega_{i}=1,
$$

or

$$
\underset{\omega}{\operatorname{Minimize}} \sum_{i=1}^{n} \sum_{j=1}^{n} \omega_{i} \omega_{j} \Sigma_{i j B} \quad \text { subject to } \sum_{i=1}^{n} \omega_{i} \mu_{i}=E_{*} \text { and } \sum_{i=1}^{n} \omega_{i}=1,
$$

where $\Sigma_{i j B}, i=1, \ldots, n ; j=1, \ldots, n$, are the elements of the Semicovariance matrix of the portfolio.

If they are exogenous, then the optimization problem (3) (or (4)) could be formally solved. The weights that minimize the Semivariance could be then obtained in the same manner as in the Mean-Variance framework. Unfortunately, this is not the case and finding the portfolio with the minimum Downside Risk is not straightforward. The main hurdle is that the Semicovariance matrix is endogenous, that is a change in weights affects the periods in which the portfolio under-performs the target rate of return, which in turn affects the elements of the Semivariance matrix.

In order to estimate the portfolio Semivariance, Markowitz (1959) suggests the following expression for $\Sigma_{i j B}$ :

$$
\Sigma_{i j B}=\frac{1}{T} \sum_{t \in V}\left(r_{i t}-B\right)\left(r_{j t}-B\right)
$$

where $V$ is a set of indices where the portfolio under performs the target return $B$.

This estimator has one advantage and one drawback. Althought it provides an exact estimation of the portfolio Semivariance, the Semicovariance matrix is still endogenous.

Many authors propose other ways to estimate the Semivariance and resolve problem (3). Among them, Hogan and Warren (1974) propose to use the Frank-Wolf algorithm. Ang (1975) proposes to linearize the Semivariance so that the optimization problem can be solved using linear programming. Harlow (1991) also considers problem (3) and generates Mean-Semivariance efficient frontier, which he compares to the Mean-Variance efficient frontier. Mamoghli and Daboussi (2010) improve Harlow approach. Their model permits to surmount the problem of inequality of the Cosemivariance measures which occur in the Mean-Semivariance model of Harlow. Markowitz et al (1993) transform the Mean-Semivariance problem into a quadratic problem by adding fictitious securities. Estrada (2008) proposes a simple and accurate heuristic approach that yields a symmetric and exogenous Semicovariance matrix, which enables the determination of Mean-Semivariance optimal portfolios by using the well-known closed-form solutions of MeanVariance problems. Athayde (2003) generalizes his own iterative algorithm developed in (2001) to construct a MeanDownSide Risk portfolio frontier.

The subsection hereafter deals with Athayde's algorithm (2001) that solves Problem (3) using an iterative procedure. 


\subsection{Parametric Athayde's Algorithm}

Suppose they are $n$ assets and that $R_{j t}$ denotes the excess return of asset $j$ at time $t$, that is $R_{j t}=r_{j t}-B$ where $B$ is the chosen benchmark. Let $M$ be the matrix with coefficients $\Sigma_{i j B}=\frac{1}{T} \sum_{t=1}^{V} R_{i t} R_{j t}$, where periods 1 through $V$ are periods in which the portfolio under performs the target return $B$.

The optimization problem (3) can be matricially written

$$
\underset{\omega}{\arg \min } \omega^{t} M \omega \quad \text { with } \omega^{t} \mathbf{1}=1
$$

where $\omega^{t}$ is the transpose of $\omega$ and $\mathbf{1}$ is a vector of 1 . The optimization algorithm is the following

Step 1 Let $\omega_{0}$ be a starting portfolio vector weights where $\omega_{0}=\left(\omega_{0,1}, \ldots, \omega_{0, n}\right) . S_{0}$ is the set of observations in which this portfolio $\omega_{0}$ had negative deviations. $M_{0}$ is a positive semidefinite matrix where

$$
M_{0}=\frac{1}{T} \sum_{t \in S_{0}}\left[\begin{array}{c}
R_{1 t} \\
R_{2 t} \\
\vdots \\
R_{n t}
\end{array}\right]\left[\begin{array}{llll}
R_{1 t} & R_{2 t} & \cdots & R_{n t}
\end{array}\right]=\frac{1}{T} \sum_{t \in S_{0}}\left[\begin{array}{ccc}
R_{1 t}^{2} & R_{1 t} R_{2 t} & R_{1 t} R_{n t} \\
R_{2 t} R_{1 t} & R_{2 t}^{2} & R_{2 t} R_{n t} \\
\vdots & \vdots & \vdots \\
R_{n t} R_{1 t} & R_{n t} R_{2 t} & R_{n t}^{2}
\end{array}\right]
$$

Step 2 Find the portfolio vector weights $\omega_{1}$ that solves the following problem

$$
\underset{\omega}{\arg \min } \omega^{t} M_{0} \omega, \quad \text { with } \omega^{t} \mathbf{1}=1 .
$$

Using Lagrangian Method, the solution of this problem is

$$
\omega_{1}=\frac{M_{0}^{-1} \mathbf{1}}{\mathbf{1}^{t} M_{0}^{-1} \mathbf{1}}
$$

With the new portfolio vector weights $\omega_{1}$, the set $S_{1}$ of indices of only negative excess returns is then formed.

If $M_{1}$ is non-invertible, it means that only a few observations are available, and that it will be possible to find a portfolio that will give a null DSR (for example when $R_{j t} \geq 0$ ). It does not mean there is no DSR, it only means that the sample in question is poor, leaving us with few degrees of freedom.

Step 3 Form a new positive semidefinite matrix $M_{1}$ where

$$
M_{1}=\frac{1}{T} \sum_{t \in S_{1}}\left[\begin{array}{ccc}
R_{1 t}^{2} & R_{1 t} R_{2 t} & R_{1 t} R_{n t} \\
R_{2 t} R_{1 t} & R_{2 t}^{2} & R_{2 t} R_{n t} \\
\vdots & \vdots & \vdots \\
R_{n t} R_{1 t} & R_{n t} R_{2 t} & R_{n t}^{2}
\end{array}\right]
$$

The next step is to find the portfolio vector weights $\omega_{2}$ that solves the following problem:

$$
\underset{\omega}{\arg \min } \omega^{t} M_{1} \omega, \quad \text { such that } \omega^{t} \mathbf{1}=1
$$

As previously, the solution to this problem is

$$
\omega_{2}=\frac{M_{1}^{-1} \mathbf{1}}{\mathbf{1}^{t} M_{1}^{-1} \mathbf{1}}
$$

Step 4 Iterate the previous process to construct a sequence of matrices $M_{i}$ until getting the first matrix $M^{F}$ satisfying the criterion $M_{F}=M_{F+1}$. Optimal portfolio vector weights is then given by:

$$
\omega_{F}=\frac{M_{F}^{-1} \mathbf{1}}{\mathbf{1}^{t} M_{F}^{-1} \mathbf{1}} .
$$

This optimal vector gives the minimum DSR. 


\subsection{Mean DSR Frontier}

In order to build the portfolio frontier, other points on the efficient set should be found. Let $\mu=\left(\mu_{1}, \ldots, \mu_{n}\right)$ be the mean vector of the assets returns., i.e $\mu_{j}$ is the mean return of asset $j, j=1, \ldots, n$ and $r_{p t}=\omega_{1} r_{1 t}+\cdots+\omega_{n t} r_{n t}$ where $r_{j t}$ is the return of the asset $j$ on time $t$. An expected return $E_{*}$ should be fixed in order to solve the new recursive minimization problem

$$
\underset{\omega}{\arg \min } \frac{1}{T} \sum_{t=1}^{T} \min \left(r_{p t}-B, 0\right)^{2}, \quad \text { i.e., } \underset{\omega}{\arg \min } \omega^{t} M \omega,
$$

subject to $(A): \omega^{t} \mu=E_{*}$ and $(B): \omega^{t} \mathbf{1}=1$.

Remark. (A) requires the weighted returns of the assets to be higher than the expected portfolio return and (B) eliminates idle investment capitals by equating the total portfolio weight to one.

Following the same recursive minimization procedure and the Lagrangian Method optimisation, the convergence is achieved after $F$ iterations and the optimal portfolio will be

$$
\omega_{F}=\frac{\alpha E_{*}-\lambda}{\alpha \theta-\lambda^{2}} M_{F}^{-1} \mu+\frac{\theta-\lambda E_{*}}{\alpha \theta-\lambda^{2}} M_{F}^{-1} \mathbf{1}
$$

where $\alpha=\mathbf{1}^{t} M_{F}^{-1} \mathbf{1}, \lambda=\mu^{t} M_{F}^{-1} \mathbf{1}$ et $\theta=\mu^{t} M_{F}^{-1} \mu$.

It should be noted that for small changes in the expected return, the matrix will remain unchanged. Pre-multiplying $\omega_{F}^{t} M_{F}$ by $[11$, , we found

$$
\operatorname{DSR}\left(\omega_{F}\right)=\frac{\alpha\left(E_{*}\right)^{2}-2 \lambda E_{*}+\theta}{\alpha \theta-\lambda^{2}}
$$

Remark. It is clear that there are no constraints on the signs of weights $\omega_{F, 1}, \ldots, \omega_{F, n}$ which make up the vector $\omega$. We will introduce them in next sections. This is one of the contributions that makes our work different from Athayde's (2003).

\section{Nonparametric Mean-Downside Risk Model}

In practice, only a finite number of observations are available, and although a large number of data is available, the assumptions of asset returns being identically distributed during the whole period of the sample may not be a very realistic assumption. The idea behind this section is to make use of nonparametric techniques to estimate continuous distributions of the portfolios, and from these estimations, optimize their DSR, constructing with this, a new portfolio frontier.

The first meaning of nonparametric covers techniques that do not rely on data belonging to any particular distribution. In particular, they may be applied in situations where less is known about the application in question.

\subsection{Basic Background}

In the following, estimate of the density of the returns using kernels is proposed. A kernel estimation of one point can be seen as a weighted average of the observations, in which the weight given to each observation decreases with its distance from the point in question.

First of all, let us introduce some elements which will be used to define kernel estimators:

The kernel $K$ It is a probability density function. For theoretical commodity, $K$ is supposed satisfying the following properties

$$
\int z K(z) d z=0, \quad \int z^{2} K(z) d z<\infty .
$$

Examples:

- Rectangular : $K(z)=\frac{1}{2}$ for $|z|<1,0$ otherwise.

- Triangular: $K(z)=1-|z|$ for $|z|<1,0$ otherwise. 
- Biweight : $K(z)=\frac{15}{16}\left(1-z^{2}\right)^{2}$ for $|z|<1,0$ otherwise.

- Epanechnikov: $K(z)=\frac{3}{4}\left(1-\frac{1}{5} z^{2}\right)$ for $|z|<\frac{1}{5}, 0$ otherwise.

- Gaussian : $K(z)=\frac{1}{\sqrt{2 \pi}} \exp \left(-\frac{1}{2} z^{2}\right)$.

The bandwidth $h(T)$ It is a positive sequence such that $h(T) \rightarrow 0$ as $T \rightarrow \infty$. It is also called the the smoothing parameter.

\subsection{Kernel Mean Return Estimation}

To build a more sophisticated estimation of DSR, Athayde $(2001,2003)$ proposed to replace all the observations $r_{t}$, $t=1, \ldots, T$ by their Mean Kernel Estimation, Mean Regression, $\hat{r}_{t}, t=1, \ldots, T$ which are a weighted average of the observations, in which the weight given to each observation decreases with its distance from the point in question.

The estimator $\hat{r}_{t}$ of the return $r_{t}$ for a given asset or portfolio is then given by

$$
\hat{r}_{t}=\frac{\sum_{l=1}^{T} r_{l} K\left(\frac{r_{t}-r_{l}}{h}\right)}{\sum_{l=1}^{T} K\left(\frac{r_{t}-r_{l}}{h}\right)},
$$

or

$$
\hat{r}_{t}=\underset{z \in \mathbb{R}}{\arg \min } \frac{\sum_{l=1}^{T}\left|r_{l}-z\right|^{2} K\left(\frac{r_{t}-r_{l}}{h}\right)}{\sum_{l=1}^{T} K\left(\frac{r_{t}-r_{l}}{h}\right)} .
$$

The density $K$ determines the shape of the weighting function. The bandwidth, $h$ determines the width of the weighting function and hence the amount of smoothing. The two components determine the properties of the estimator. Theoretical (minimization of asymptotic errors) and practical (rule of thumb, cross validation, plug-in methods) researches had been carried out (and continues to be carried out) on the question of how one should select $K$ and $h$ in order to optimize the properties of the estimator. More details can be found in Pagan and Ullah (1999) or in Silverman (1986).

Remark. In his paper, Athayde (2003) used the same weights for the estimation of portfolio returns as for the assets' ones. He started by the estimation of the portfolio returns, then, he derived the returns estimation for each asset. This paper presents another alternative.

\subsection{Kernel Median Return Estimation}

\subsubsection{General Concept}

The disadvantage of the above Mean Regression is that it is sensitive to outliers and may be inappropriate in some cases, as when the distribution is multi-modal or highly asymmetric. This problem can be solved by using another useful descriptive statistic which is robust to heavy-tailed error distributions and outliers: the Median Regression. It is a more complete picture of the distribution than the one given by its mean.

From a theoretical point on view, if $(X, Z)$ represents a $\mathbb{R}^{2}$-valued random vector, the conditional median, $q(x)$, of $Z$ given $X=x$ is the solution of the following minimization problem

$$
q(x)=\underset{y \in \mathbb{R}}{\arg \min } \int|z-y| F(d z \mid X=x) .
$$

It is easy to get an estimator $\hat{q}(x)$ of $q(x)$ by replacing $F(d z \mid X=x)$ by an appropriate estimator $F_{T}(\cdot \mid X=x)$. Then

$$
\hat{q}(x)=\underset{y \in \mathbb{R}}{\arg \min } \int|z-y| F_{T}(d z \mid X=x) .
$$

More details on theoretical and applied properties of median (and more generally on quantiles) can be found in Berlinet et al (2001) or Gannoun et al (2003).

Remark. 1. The conditional distribution function can be seen as a conditional expectation i.e. $\mathbb{E}\left(1_{\{Z \leq z\}} \mid X=x\right)=$ $F(z \mid X=x)$. 
2. If $F(\cdot \mid X=x)$ is continuous and strictly increasing, the conditional median of $Z$ given $X=x$ is obtained by the following

$$
q(x)=F^{-1}(0.5 \mid X=x) .
$$

\subsubsection{Kernel Median Estimators}

Given $T$ observations $r_{t}, t=1, \ldots, T$, from a given asset or portfolio, the kernel estimator $F_{T}\left(z \mid r_{t}\right)$ of the conditional distribution function $F\left(z \mid r_{t}\right)$ is defined by

$$
F_{T}\left(z \mid r_{t}\right)=\frac{\sum_{l=1}^{T} 1_{\left\{r_{l} \leq z\right\}} K\left(\frac{r_{t}-r_{l}}{h}\right)}{\sum_{l=1}^{T} K\left(\frac{r_{t}-r_{l}}{h}\right)}
$$

Using Koenker (2005) and (16), we can get directly a kernel median return estimation as follows

$$
\hat{r}_{t}=\underset{z \in \mathbb{R}}{\arg \min } \frac{\sum_{l=1}^{T}\left|r_{l}-z\right| K\left(\frac{r_{t}-r_{l}}{h}\right)}{\sum_{l=1}^{T} K\left(\frac{r_{t}-r_{l}}{h}\right)} .
$$

Another way to get $\hat{r}_{t}$ is to solve the following equation

$$
F_{T}\left(z \mid r_{t}\right)=\frac{\sum_{l=1}^{T} 1_{\left\{r_{l} \leq z\right\}} K\left(\frac{r_{t}-r_{l}}{h}\right)}{\sum_{l=1}^{T} K\left(\frac{r_{t}-r_{l}}{h}\right)}=\frac{1}{2}
$$

Remark. 1. To compute $\hat{r}_{t}$, the function $\mathbf{n l m}$, from the R package stats, is considered $h t t p s: / /$ stat.ethz.ch/R-manual/Rdevel/library/stats/html/nlm.html

2. In the statistical literature, they are more complicated nonparametric estimators of conditional median : Local polynomial method, double kernel method, L-estimator method, ... (see Gannoun et al (2002) fore more details).

3. Median smooths better than mean.

4. Empirically, it is less sensitive than the mean.

\subsubsection{The Kernel DSR Estimation}

The portfolio return estimators are derived from those of Kernel nonparametric assets ones. Let us recall these estimators:

mean estimator $\hat{r}_{j t}=\frac{\sum_{l=1}^{T} r_{j l} K\left(\frac{r_{j i t}-r_{j l}}{h}\right)}{\sum_{l=1}^{T} K\left(\frac{r_{j i t}-r_{j l}}{h}\right)}$

median estimator $\hat{\hat{r}}_{j t}=\underset{z \in \mathbb{R}}{\arg \min } \frac{\sum_{l=1}^{T}\left|r_{j l}-z\right| K\left(\frac{{ }^{r}{ }^{\prime}-r_{j l}}{h}\right)}{\sum_{l=1}^{T} K\left(\frac{r_{j i t} r_{j l}}{h}\right)}$

for each asset $j$ on time $t, j=1, \ldots, n$ and $t=1, \ldots, T$.

If $\omega_{j}$ is the percentage weight of the $j$ th asset in the portfolio, the return on each time $t$ is given by $r_{p t}=\omega_{1} r_{1 t}+$ $\cdots+\omega_{n} r_{n t}$ and its estimation is given by $\tilde{r}_{p t}=\omega_{1} \tilde{r}_{1 t}+\cdots+\omega_{n} \tilde{r}_{n t}$ where $\tilde{r}_{j t}=\hat{r}_{j t}$ or $\hat{\hat{r}}_{j t}$. then

To get a kernel DSR estimation, we replace in (2) all the observations $r_{p t}, t=1, \ldots, T$ by their estimators $\tilde{r}_{p t}$ and,

$$
\widetilde{D S R}(\omega)=\frac{1}{T} \sum_{t=1}^{T} \min \left(\tilde{r}_{p t}-B, 0\right)^{2} .
$$

Suppose we have $n$ assets and denote by $\tilde{R}_{j t}$ the excess return of asset $j$ at time $t$. That is $\tilde{R}_{j t}=\tilde{r}_{j t}-B$ where $B$ is the chosen benchmark. Let $\tilde{M}$ the matrix with coefficients $\Sigma_{i j B}=\frac{1}{T} \sum_{t=1}^{V} \tilde{R}_{i t} \tilde{R}_{j t}$ where $V$ is the period in which the portfolio under-performs the target return $B$. 
The optimization of assets portfolio can be modelled as follows

$$
\operatorname{minimize} \frac{1}{T} \sum_{t=1}^{T} \min \left(\tilde{r}_{p t}-B, 0\right)^{2}, \quad \text { i.e., minimize } \omega^{t} \tilde{M} \omega \text {, }
$$

subject to $(A): \omega^{t} \mu=E_{*},(B): \omega^{t} \mathbf{1}=1$ and $(C): \omega_{j} \geq 0, j=1, \ldots, n$.

The constraint (C) restricts any short selling of assets.

\subsubsection{Kernel DSR Minimization Algorithm}

The processing is similar to the classical one. The estimated returns $\tilde{r}_{j t}$ will take place of the original returns in the previous algorithm. $\tilde{\mu}_{j}$ denotes $\overline{\tilde{r}}_{j}, j=1, \ldots, n$ and $E_{*}$ is a given expected return.

\section{With short sellig constraint (without the constraint $(\mathrm{C})$ ).}

We are facing to the following ooptimization problem:

$$
\text { minimize } \omega^{t} \tilde{M} \omega \text { subject to }(A): \omega^{t} \tilde{\mu}=E_{*} \text { and }(B): \omega^{t} \mathbf{1}=1 \text {. }
$$

Many steps are necessary to get the optimal solution.

Step 1 We start with an arbitrary portfolio $\omega_{0}=\left(\omega_{0,1}, \ldots, \omega_{0, n}\right)$. For each date $t, t=1, \ldots, T$, the returns of this portfolio

$$
r_{0, p t}=\omega_{0,1} r_{1 t}+\ldots+\omega_{0, n} r_{n t}
$$

will be replaced by

$$
\tilde{r}_{0, p t}=\omega_{0,1} \tilde{r}_{1 t}+\ldots+\omega_{0, n} \tilde{r}_{n t}
$$

From the previous estimators (22), we select the dates when the estimated returns of portfolio $w_{0}$ had negative excess returns. This set is called $S_{0}$. Let $\tilde{R}_{0, i t}=\tilde{r}_{i t}-B$ and $\tilde{M}_{0}$ the following positive semidefinite matrix

$$
\tilde{M}_{0}=\frac{1}{T} \sum_{t \in S_{0}}\left[\begin{array}{c}
\tilde{R}_{1 t} \\
\tilde{R}_{2 t} \\
\vdots \\
\tilde{R}_{n t}
\end{array}\right]\left[\begin{array}{llll}
\tilde{R}_{1 t} & \tilde{R}_{2 t} & \cdots & \tilde{R}_{n t}
\end{array}\right]=\frac{1}{T} \sum_{t \in S_{0}}\left[\begin{array}{ccc}
\tilde{R}_{1 t}^{2} & \tilde{R}_{1 t} \tilde{R}_{2 t} & \tilde{R}_{1 t} \tilde{R}_{n t} \\
\tilde{R}_{2 t} \tilde{R}_{1 t} & \tilde{R}_{2 t}^{2} & \tilde{R}_{2 t} \tilde{R}_{n t} \\
\vdots & \vdots & \vdots \\
\tilde{R}_{n t} \tilde{R}_{1 t} & \tilde{R}_{n t} \tilde{R}_{1 t} & \tilde{R}_{n t}^{2}
\end{array}\right]
$$

Step 2 Find the portfolio $\omega_{1}$ that minimizes $\omega^{t} \tilde{M}_{0} \omega$ subject to $(A): \omega^{t} \tilde{\mu}=E_{*}$ and $(B): \omega^{t} \mathbf{1}=1$. Using Lagrangian method, the solution is the following

$$
\omega_{1}=\frac{\alpha_{0} E_{*}-\lambda_{0}}{\alpha_{0} \theta-\lambda_{0}^{2}} \tilde{M}_{0}^{-1} \tilde{\mu}+\frac{\theta_{0}-\lambda_{0} E_{*}}{\alpha_{0} \theta_{0}-\lambda_{0}^{2}} \tilde{M}_{0}^{-1} \mathbf{1}
$$

where $\alpha_{0}=\mathbf{1}^{t} \tilde{M}_{0}^{-1} \mathbf{1}, \lambda_{0}=\tilde{\mu}^{t} \tilde{M}_{0}^{-1} \mathbf{1}$ et $\theta_{0}=\tilde{\mu}^{t} \tilde{M}_{0}^{-1} \mu$.

Step 3 Using 23, we calculate the new returns of the portfolio

$$
\tilde{r}_{1, p t}=\omega_{1,1} \tilde{r}_{1 t}+\ldots+\omega_{1, n} \tilde{r}_{n t}
$$

and we construct $S_{1}$ the set all indices such that $\tilde{r}_{1, p t}-B<0$, i.e., $S_{1}=\left\{t \in\{1, \ldots, T\}: \tilde{r}_{1, p t}-B<0\right\}$. 
Using the above estimators, we built the following new positive definite matrix $\tilde{M}_{1}$

$$
\begin{aligned}
\tilde{M}_{1} & =\frac{1}{T} \sum_{t \in S_{1}}\left[\begin{array}{c}
\tilde{R}_{1 t} \\
\tilde{R}_{2 t} \\
\vdots \\
\tilde{R}_{n t}
\end{array}\right]\left[\begin{array}{llll}
\tilde{R}_{1 t} & \tilde{R}_{2 t} & \cdots & \tilde{R}_{n t}
\end{array}\right] \\
& =\frac{1}{T} \sum_{t \in S_{1}}\left[\begin{array}{ccc}
\left(\tilde{R}_{1 t}\right)^{2} & \tilde{R}_{1 t} \tilde{R}_{2 t} & \tilde{R}_{1 t} \tilde{R}_{n t} \\
\tilde{R}_{2 t} \tilde{R}_{1 t} & \left(\tilde{R}_{2 t}\right)^{2} & \tilde{R}_{2 t} \tilde{R}_{n t} \\
\vdots & \vdots & \vdots \\
\tilde{R}_{n t} \tilde{R}_{1 t} & \tilde{R}_{n t} \tilde{R}_{1 t} & \left(\tilde{R}_{n t}\right)^{2}
\end{array}\right]
\end{aligned}
$$

Using Lagrangian method, the solution is the following

$$
\omega_{2}=\frac{\alpha_{1} E_{*}-\lambda_{1}}{\alpha_{1} \theta_{1}-\lambda_{1}^{2}} \tilde{M}_{1}^{-1} \tilde{\mu}+\frac{\theta_{1}-\lambda_{1} E_{*}}{\alpha_{1} \theta_{1}-\lambda_{1}^{2}} \tilde{M}_{1}^{-1} \mathbf{1}
$$

where $\alpha_{1}=\mathbf{1}^{t} \tilde{M}_{1}^{-1} \mathbf{1}, \lambda_{1}=\tilde{\mu}^{t} \tilde{M}_{1}^{-1} \mathbf{1}$ et $\theta_{1}=\tilde{\mu}^{t} \tilde{M}_{1}^{-1} \mu$.

Step 4 Again, find a new portfolio, select its negative observations, construct a matrix $\tilde{M}_{2}$, repeat the minimization with $\tilde{M}_{2}$, find a new portfolio, select its negative deviations, and so on. The iterations will stop when the matrix $\tilde{M}_{F}$ will be the same as $\tilde{M}_{F+1}$. The minimum DSR portfolio with expected return $E_{*}$ is given by

$$
\omega_{F+1}=\frac{\alpha_{F} E_{*}-\lambda_{F}}{\alpha_{F} \theta_{F}-\lambda_{F}^{2}} \tilde{M}_{F}^{-1} \tilde{\mu}+\frac{\theta_{F}-\lambda_{F} E_{*}}{\alpha_{F} \theta_{F}-\lambda_{F}^{2}} \tilde{M}_{F}^{-1} \mathbf{1}
$$

where $\alpha_{F}=\mathbf{1}^{t} \tilde{M}_{F}^{-1} \mathbf{1}, \lambda_{F}=\tilde{\mu}^{t} \tilde{M}_{F}^{-1} \mathbf{1}$ et $\theta_{F}=\tilde{\mu}^{t} \tilde{M}_{F}^{-1} \tilde{\mu}$.

The Downside Risk is then

$$
\operatorname{DSR}\left(\omega_{F+1}\right)=\frac{\alpha_{F} E_{*}^{2}-2 \lambda_{F} E_{*}+\theta_{F}}{\alpha_{F} \theta_{F}-\lambda_{F}^{2}}
$$

\section{Without short selling constraint (with the Constraint (C)).}

The optimization problem consists in minimizing

$$
\omega^{t} \tilde{M} \omega \text { subject to }(A): \sum_{j=1}^{n} \omega_{j} \tilde{\mu}_{j}=E_{*},(B): \sum_{j=1}^{n} \omega_{j}=1 \text { and }(C): \omega_{j} \geq 0, j=1, \ldots, n
$$

All the minimization steps are similar to the previous ones. Nevertheless, the intermediate optimized solution on each step is not explicit. To get them, we use an appropriated software located in $\mathbf{R}$ Tool Box with adapted inputs : quadrog (http://cran.r-project.org/web/packages/quadprog/).

Remark. - From a computationally point of view, there are less iterations than in Ben Salah (2014) where , the estimation of all returns are necessary in each step. The convergence is achieved after finite number of iterations.

- In all his minimization model, Athayde never took into account the $(\mathrm{C})$ constraint. Since these constraint is accounted for in this paper, our results are slightly different from his.

The DSR is then given by

$$
\operatorname{DSR}\left(\omega_{F+1}\right)=\omega_{F+1}^{t} \tilde{M}_{F} \omega_{F+1} .
$$

In order to build the portfolio frontier, we will have to find the DSR for varied expected return $E_{*}$. For a given $E_{*}$, the same procedure as in previous paragraphs is used to determine the DSR of the optimal portfolio. The problem is computationally less complicated than in Ben Salah et al (2015) since we have not new estimations of returns for every assets at each optimization step. 


\section{Empirical Analysis}

This section is devoted to investigate the performance of the proposed methods using both the parametric and nonparametric approaches on two different stock markets (emergent and developed one). This empirical study will be made in two cases: with and without short selling constraints.

\subsection{Data}

A dataset, drawn from Reuters, was used for this analysis. The original Data consists in daily 9 stocks returns belonged to different sectors: banks, insurance, industry, energy, technology and telecommunication, from 2 markets:

- the French stock market (as a developed country) throughout the period from April 2000 to April 2014, yielding a total of 3579 daily observations,

- the Brazilian stock market (as an emergent country) throughout the period from October 2009 to December 2013, yielding a total of 1018 daily observations.

CAC 40 (the French Stock Market Index) and BOVESPA (Brasil Sao Paulo Stock Exchange Index) values (observed in the respective periods) will provide a baseline for subsequent comparisons between the different methods.

The Government French Bonds, from for January the 2nd, 2013 to April the 26th, 2014), and the one year Government Brazilian Bond (2012) are considered as the Risk-Free Rate of returns in order to compute some Indexes to measure and compare the performance of the different methods.

In optimization procedures:

- the benchmark B is supposed to be equal to zero,

- the kernel $K$ is the Gaussian density,

- the bandwidth $h$ is chosen by cross validation method.

Historical Price Data $p_{t}$ is adjusted for dividends. The assets returns are computed from stock prices observed on Thomson Reuters Platform as follows:

$$
R_{t}=\frac{p_{t}-p_{t-1}}{p_{t-1}} \text {, with } p_{t}: \text { Stock closing price at date } t \text { and } p_{t-1}: \text { Stock closing price at date } t-1
$$

Remark. The choose of the kernel is not crucial in nonparametric estimation. Indeed, different densities give slightly the same results. However, the bandwidth selection is very important in order to improve the quality of estimation. The choose of cross validation rule to determine the bandwidth is motivated by its popularity in nonparametric literature (see Arlot and Celisse (2010)). 
Table 1: French Market Historical Statistics

\begin{tabular}{lcccccc}
\hline & Min & Mean & sd & Skewness & Kurtosis & Max \\
\hline Accor & -0.1294 & 0.000225 & 0.02230 & 0.09481 & 3.0857 & 0.1241 \\
Airb & -0.2632 & 0.000436 & 0.02580 & -0.13740 & 5.5292 & 0.1340 \\
Alcat & -0.1975 & 0.000531 & 0.03607 & 0.45715 & 8.4942 & 0.4054 \\
BNP & -0.1724 & 0.000327 & 0.02601 & 0.65718 & 8.8503 & 0.2090 \\
Carr & -0.1102 & 0.000157 & 0.01977 & 0.12020 & 2.8544 & 0.0995 \\
Dano & -0.1050 & 0.000285 & 0.01571 & 0.09250 & 4.2764 & 0.1019 \\
Oréal & -0.1112 & 0.000261 & 0.01745 & 0.21990 & 4.5650 & 0.1474 \\
S.Gén & -0.1623 & 0.000251 & 0.02890 & 0.39010 & 6.7635 & 0.2389 \\
Total & -0.0919 & 0.0001431 & 0.01716 & 0.22629 & 5.6594 & 0.1364 \\
\hline
\end{tabular}

Table 2: Brazilian Descriptive Market

\begin{tabular}{lcccccc}
\hline & Min & Mean & S.D & Skewness & Kurtosis & Max \\
\hline B. Ameri. & -0.2954 & 0.00017 & 0.02839 & 0.3754 & 29.479 & 0.2643 \\
B..Estado & 0.0882 & 0.00014 & 0.02509 & 1.0275 & 7.121 & 0.2052 \\
Pet.e.Gas & -0.2533 & -0.00087 & 0.03237 & -0.9176 & 8.528 & 0.1454 \\
Cosan.Ltd & -0.1339 & -0.00132 & 0.02341 & -0.2088 & 2.361 & 0.1025 \\
Informatica & -0.1555 & -0.00127 & 0.02687 & -0.4985 & 5.901 & 0.1560 \\
Ideiasnet & -0.0120 & -0.00097 & 0.02624 & 0.0792 & 2.524 & 0.1357 \\
Serv. Enge & -0.1468 & -0.000268 & 0.03143 & 0.7219 & 9.067 & 0.2758 \\
Gol.Intel. & -0.0216 & 0.000147 & 0.03171 & 0.1646 & 5.093 & 0.1776 \\
Oi.SA & -0.1084 & 0.000000 & 0.02133 & 0.1068 & 1.740 & 0.0946 \\
\hline
\end{tabular}

\section{Historical statistics:}

In term of the third moment of expected returns (Skewness), the returns of French assets Alcatel, BNP and Société Générale are highly positive skewed whereas the return of the Airbus asset is negative skewed. Carrefour, L'Oréal and Total returns assets are moderately skewed. However, returns distribution of the assets Accor and Danone are approximatively normal. The returns of Brazilian assets Banco Pan Americano, Banco de Estado de Sergipe, SA Ideiasnet, Estruturas e servicos de Engenharia, Gol Linhas aereas Inteligente and Oi SA have a positive skew whereas the returns of assets Petroleo e Gas, Cosan Ltd and Positivo Informatica have a negative one. For the assets Banco de Estado de Sergipe and Petroleo e Gas, the skewness is substantial and the distribution is far from symmetrical. Only the return distribution of SA Ideiasnet can be considered Gaussian. All the other distributions assets have a considerable positive skew. For the fourth moment of expected returns (Kurtosis), no relevant fat-tailed distribution is observed in French Market. All the distributions are more peaked than the Gaussian distribution. In the brazilian case, all the distributions are peaked than the Gaussian one. Significant fat-tails are observed for returns distribution of the assets Banco Pan Americano, Petroleo e Gas, Estruturas e servicos de Engenharia and Banco de Estado de Sergipe (see Table 1 and Table 2).

Therefore, these findings suggest the use of the DownSide Risk methods to find the optimal portfolio.

The correlation matrix indicates that the returns are correlated between each other in the two stock markets (see Table 3 and Table 4 . 
Table 3: French Correlation Matrix

$\begin{array}{lccccccccc} & \text { Accor } & \text { Airb } & \text { Alcat } & \text { BNP } & \text { Carr } & \text { Dano } & \text { Oréal } & \text { S. Gén } & \text { Total } \\ \text { Accor } & 1.000 & 0.457 & 0.447 & 0.544 & 0.474 & 0.351 & 0.426 & 0.539 & 0.489 \\ \text { Airb } & 0.457 & 1.000 & 0.401 & 0.418 & 0.404 & 0.337 & 0.376 & 0.407 & 0.415 \\ \text { Alcat } & 0.447 & 0.401 & 1.000 & 0.465 & 0.422 & 0.28 & 0.383 & 0.476 & 0.404 \\ \text { BNP } & 0.544 & 0.418 & 0.465 & 1.000 & 0.522 & 0.367 & 0.469 & 0.803 & 0.554 \\ \text { Carr } & 0.474 & 0.404 & 0.422 & 0.522 & 1.000 & 0.454 & 0.518 & 0.508 & 0.527 \\ \text { Dano } & 0.351 & 0.337 & 0.28 & 0.367 & 0.454 & 1.000 & 0.488 & 0.347 & 0.455 \\ \text { Oréal } & 0.426 & 0.376 & 0.383 & 0.469 & 0.518 & 0.488 & 1.000 & 0.434 & 0.512 \\ \text { S. Gén } & 0.539 & 0.407 & 0.476 & 0.803 & 0.508 & 0.347 & 0.434 & 1.000 & 0.514 \\ \text { Total } & 0.489 & 0.415 & 0.404 & 0.554 & 0.527 & 0.455 & 0.512 & 0.514 & 1.000\end{array}$

Table 4: Brazilian Correlation Matrix

$\begin{array}{lccccccccc} & \text { B.Am } & \text { B.Es } & \text { P.e.G } & \text { Co.Lt } & \text { Info } & \text { Idei } & \text { Se.Eng } & \text { Go.in } & \text { Oi.Sa } \\ \text { B.Am } & 1.000 & 0.031 & 0.196 & 0.039 & 0.134 & 0.113 & 0.149 & 0.203 & 0.03 \\ \text { B.Es } & 0.031 & 1.000 & 0.014 & -0.003 & -0.074 & -0.026 & 0.007 & 0.014 & -0.031 \\ \text { P. e G. } & 0.196 & 0.014 & 1.000 & 0.073 & 0.23 & 0.23 & 0.456 & 0.396 & 0.059 \\ \text { Co.Lt } & 0.039 & -0.003 & 0.073 & 1.000 & 0.033 & 0.006 & 0.067 & 0.003 & -0.055 \\ \text { Info. } & 0.134 & -0.074 & 0.23 & 0.033 & 1.000 & 0.17 & 0.213 & 0.229 & 0.09 \\ \text { Idei } & 0.113 & -0.026 & 0.23 & 0.006 & 0.17 & 1.000 & 0.216 & 0.214 & 0.092 \\ \text { Se. Eng } & 0.149 & 0.007 & 0.456 & 0.067 & 0.213 & 0.216 & 1.000 & 0.358 & 0.036 \\ \text { Go.In } & 0.203 & 0.014 & 0.396 & 0.003 & 0.229 & 0.214 & 0.358 & 1.000 & 0.085 \\ \text { Oi.SA } & 0.03 & -0.031 & 0.059 & -0.055 & 0.090 & 0.092 & 0.036 & 0.085 & 1.000\end{array}$

\subsection{Analysis of Results}

The sample data is divided into two parts: one for making optimization and one for testing the efficiency of the methods. The same analysis is conducted on the French and Brazilian Stock Markets. The optimisation problem will be solved under two kinds of constraints: optimization without short selling (Positive Constraints on the Weights) and optimization with short selling.

The optimal portfolio returns will compared de the CAC 40 (respectively to Brazil Sao Paulo Stock Exchange Index). The performance will be measured by Sharpe and Sortino ratios.

\subsubsection{Optimization without short selling}

In this paragraph, short selling is not allowed. The suspension of short selling is generally a response to illegal practices concerning spreading false rumours in order to lower the price of shares. Another concern is the naked short selling, where investors sell shares without first having obtained the loan and then fail the settlement. In the Frensh market sample, the first $3245 \times 9$ (respectively the $783 \times 9$ in the Brazilian Market sample) assets returns are used to make the optimal portfolios. The last $334 \times 9$ (respectively $235 \times 9$ ) observations will be used for backtesting and checking the consistency of each optimization method (Markowitzc (I), Classical DSR (II), nonparametric Mean DSR Method (III), nonparametric Median DSR Method (IV)). For easy visual comparison, the curves corresponding to the efficient frontiers are plotted on the same figure (Figure 1 and Figure 2)

It is apparent from the graphical comparison in Figure 1 that the nonparametric and classical DSR methods push the efficient frontiers outward to the left of the classical Markowitz's mean variance curve to produce efficient portfolios that are stochastically dominant. In particular, for any given expected return level, the nonparametric median DSR method presents a lower risk (SSD) than the other methods. In the Brazilian case, the efficient frontier obtained by kernel median estimation dominates stochastically those obtained by the classical Mean-Variance, and by kernel mean estimation. It also appears that efficient frontiers derived from classical DSR and nonparametric median DSR 


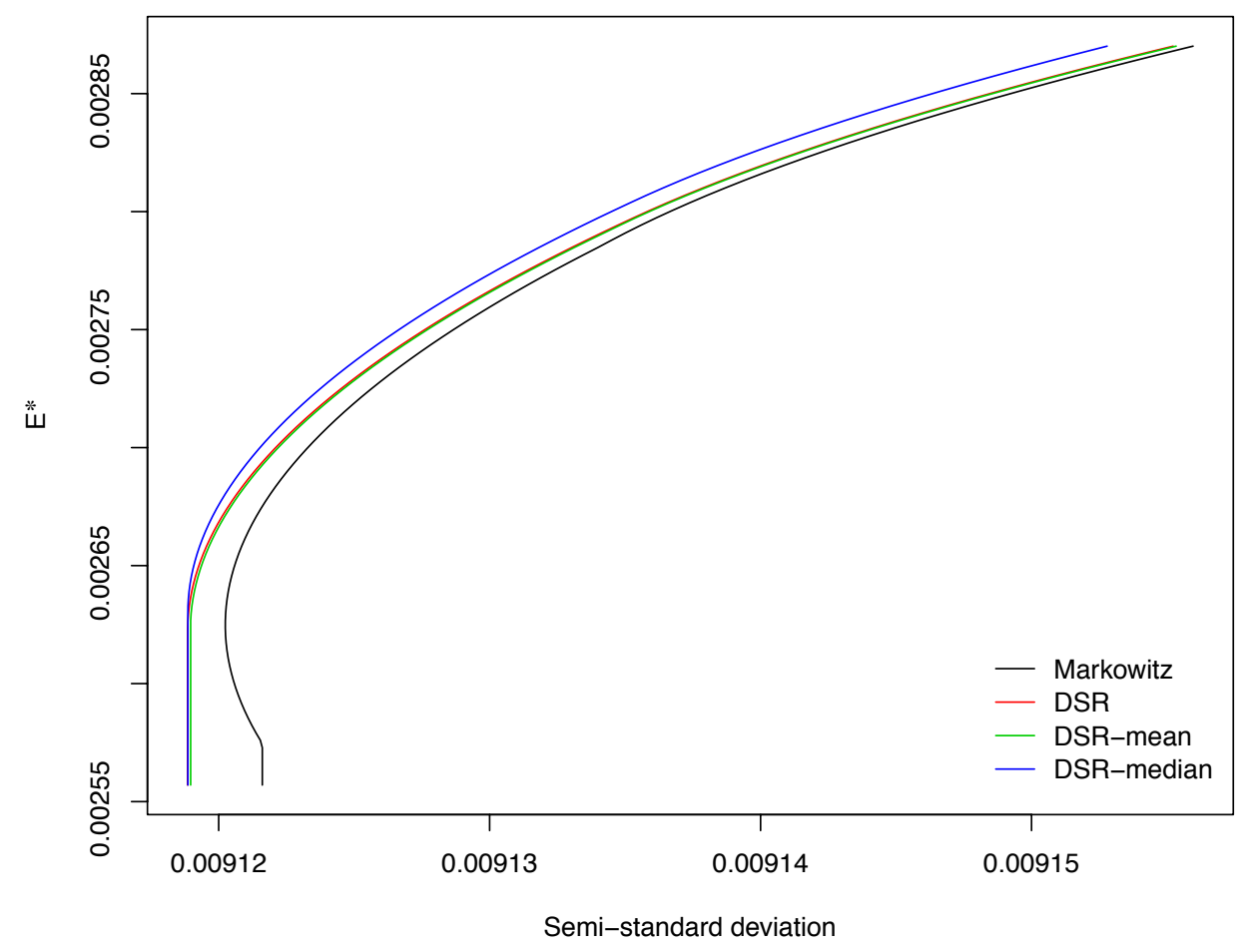

Figure 1: French Portfolio Efficient Frontiers $\left(\omega_{i} \geq 0\right)$

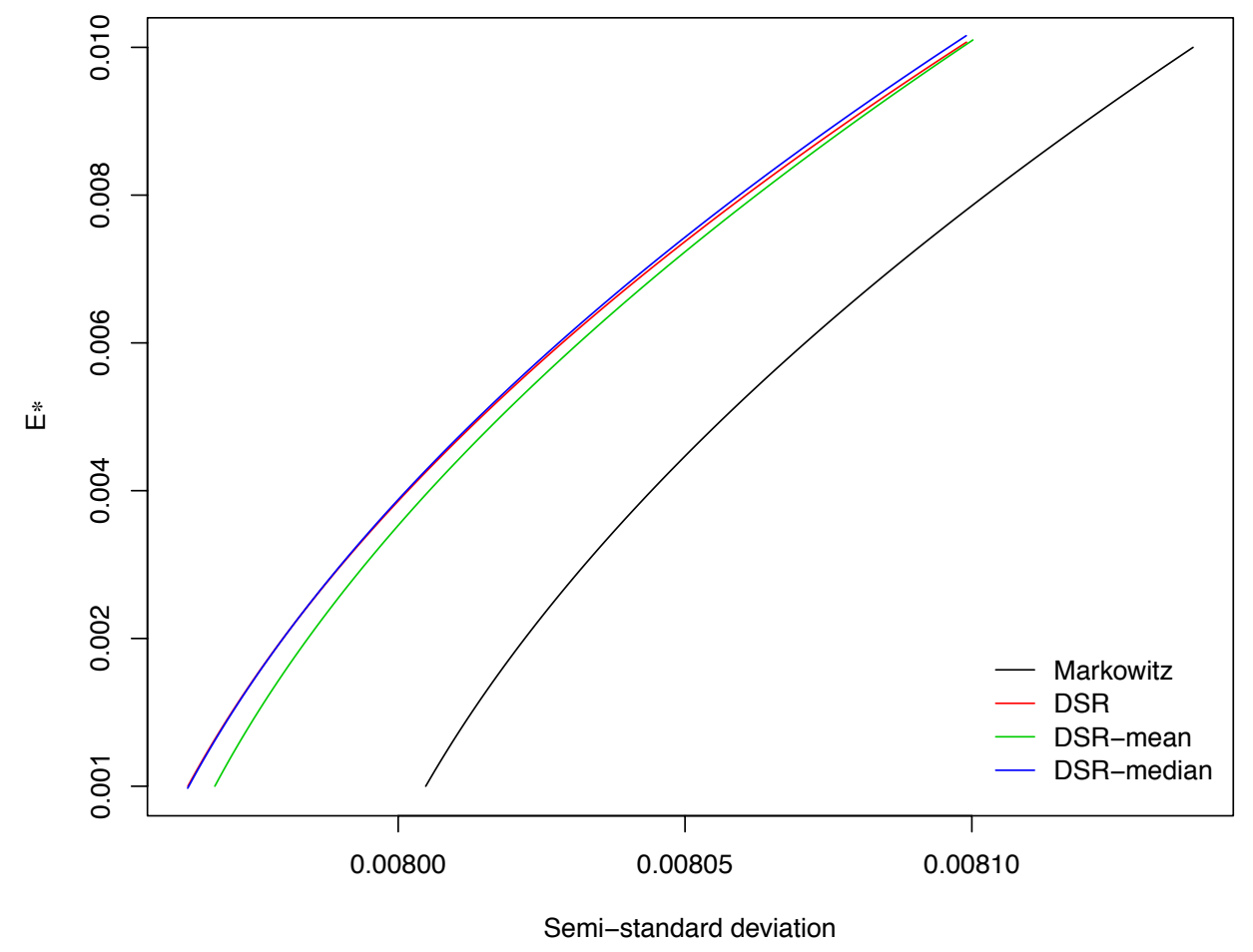

Figure 2: Brazilian Portfolio Efficient Frontiers $\left(\omega_{i} \geq 0\right)$ 
have close position and slope. These findings are confirmed by the results exhibited on Table 5 and Table 6 which give the optimal portfolios (weights) for many expected returns $E_{*}$.

Table 5: France: Optimal portfolios without short selling

\begin{tabular}{lcccccccccccc}
\hline Model & $E_{*}$ & Accor & Airb & Alcat & BNP & Carr & Dano & Oréa & S.Gle & Total & S.D. & Semi-S.D. \\
\hline I & 0.025 & 0.07 & 0.04 & 0.00 & 0.00 & 0.00 & 0.44 & 0.22 & 0.00 & 0.23 & 0.013397 & 0.009376 \\
II & 0.025 & 0.06 & 0.05 & 0.00 & 0.00 & 0.00 & 0.43 & 0.23 & 0.00 & 0.23 & 0.013396 & 0.009375 \\
III & 0.025 & 0.06 & 0.05 & 0.00 & 0.00 & 0.00 & 0.43 & 0.23 & 0.00 & 0.23 & 0.013395 & 0.009375 \\
IV & 0.025 & 0.06 & 0.04 & 0.00 & 0.00 & 0.01 & 0.43 & 0.23 & 0.00 & 0.23 & 0.013391 & 0.009373 \\
\hline I & 0.03 & 0.03 & 0.16 & 0.00 & 0.02 & 0.00 & 0.52 & 0.25 & 0.00 & 0.02 & 0.014047 & 0.009792 \\
II & 0.03 & 0.02 & 0.16 & 0.00 & 0.04 & 0.00 & 0.51 & 0.25 & 0.00 & 0.03 & 0.014044 & 0.009789 \\
III & 0.03 & 0.02 & 0.16 & 0.00 & 0.04 & 0.00 & 0.50 & 0.25 & 0.00 & 0.03 & 0.014046 & 0.009790 \\
IV & 0.03 & 0.02 & 0.15 & 0.00 & 0.03 & 0.00 & 0.51 & 0.25 & 0.00 & 0.03 & 0.013999 & 0.009761 \\
\hline I & 0.035 & 0.00 & 0.42 & 0.00 & 0.06 & 0.00 & 0.48 & 0.04 & 0.00 & 0.00 & 0.016346 & 0.011355 \\
II & 0.035 & 0.00 & 0.41 & 0.00 & 0.08 & 0.00 & 0.47 & 0.04 & 0.00 & 0.00 & 0.016341 & 0.011352 \\
III & 0.035 & 0.00 & 0.41 & 0.00 & 0.08 & 0.00 & 0.47 & 0.04 & 0.00 & 0.00 & 0.016341 & 0.011355 \\
IV & 0.035 & 0.00 & 0.40 & 0.00 & 0.06 & 0.00 & 0.50 & 0.03 & 0.00 & 0.00 & 0.016206 & 0.011263 \\
\hline I & 0.04 & 0.00 & 0.75 & 0.00 & 0.03 & 0.00 & 0.22 & 0.00 & 0.00 & 0.00 & 0.021208 & 0.014754 \\
II & 0.04 & 0.00 & 0.75 & 0.00 & 0.05 & 0.00 & 0.21 & 0.00 & 0.00 & 0.00 & 0.021205 & 0.014753 \\
III & 0.04 & 0.00 & 0.75 & 0.00 & 0.04 & 0.00 & 0.21 & 0.00 & 0.00 & 0.00 & 0.021204 & 0.014754 \\
IV & 0.04 & 0.00 & 0.75 & 0.00 & 0.01 & 0.00 & 0.24 & 0.00 & 0.00 & 0.00 & 0.021107 & 0.014691 \\
\hline
\end{tabular}

For all the used methods, Alcatel, Carrefour and Société Générale are not present in the optimal portfolios. The contribution of the Airbus and Danone assets in constitution of the optimal portfolio is significant. Accor and Total assets contribute to the optimal portfolio only for low expected return level.

Table 6: Brazil: Optimal portfolios without short selling

\begin{tabular}{lcccccccccccc}
\hline Model & $E_{*}$ & B.Am. & B.Es. & P.Ga. & Cos & Inf & Idei & S.En & G.Int. & Oi.SA & S.D. & Semi-S.D \\
\hline \hline I & 0.005 & 0.09 & 0.19 & 0.01 & 0.21 & 0.10 & 0.10 & 0.04 & 0.03 & 0.24 & 0.010780 & 0.007374 \\
II & 0.005 & 0.10 & 0.24 & 0.00 & 0.21 & 0.07 & 0.08 & 0.03 & 0.04 & 0.24 & 0.010780 & 0.007294 \\
III & 0.005 & 0.09 & 0.23 & 0.00 & 0.21 & 0.07 & 0.08 & 0.03 & 0.04 & 0.24 & 0.010774 & 0.007297 \\
IV & 0.005 & 0.10 & 0.23 & 0.00 & 0.21 & 0.07 & 0.08 & 0.03 & 0.04 & 0.24 & 0.010664 & 0.007294 \\
\hline I & 0.01 & 0.09 & 0.19 & 0.00 & 0.22 & 0.09 & 0.10 & 0.04 & 0.03 & 0.24 & 0.010780 & 0.007355 \\
II & 0.01 & 0.10 & 0.24 & 0.00 & 0.21 & 0.07 & 0.08 & 0.03 & 0.04 & 0.24 & 0.010774 & 0.007294 \\
III & 0.01 & 0.09 & 0.23 & 0.00 & 0.21 & 0.07 & 0.08 & 0.03 & 0.04 & 0.24 & 0.010763 & 0.007297 \\
IV & 0.01 & 0.10 & 0.23 & 0.00 & 0.21 & 0.07 & 0.08 & 0.03 & 0.04 & 0.24 & 0.010670 & 0.007294 \\
\hline I & 0.015 & 0.09 & 0.19 & 0.00 & 0.23 & 0.08 & 0.09 & 0.04 & 0.04 & 0.24 & 0.010787 & 0.007348 \\
II & 0.015 & 0.10 & 0.24 & 0.00 & 0.21 & 0.07 & 0.08 & 0.03 & 0.04 & 0.24 & 0.010765 & 0.007294 \\
III & 0.015 & 0.09 & 0.23 & -0.00 & 0.21 & 0.07 & 0.08 & 0.03 & 0.04 & 0.24 & 0.010783 & 0.007297 \\
IV & 0.015 & 0.10 & 0.23 & 0.00 & 0.21 & 0.07 & 0.08 & 0.03 & 0.04 & 0.24 & 0.010706 & 0.007294 \\
\hline I & 0.02 & 0.09 & 0.19 & 0.00 & 0.25 & 0.07 & 0.08 & 0.04 & 0.04 & 0.24 & 0.010775 & 0.007367 \\
II & 0.02 & 0.10 & 0.24 & 0.00 & 0.23 & 0.05 & 0.07 & 0.03 & 0.05 & 0.24 & 0.010860 & 0.007309 \\
III & 0.02 & 0.10 & 0.23 & 0.00 & 0.23 & 0.06 & 0.07 & 0.03 & 0.05 & 0.24 & 0.010837 & 0.007313 \\
IV & 0.02 & 0.10 & 0.23 & 0.00 & 0.23 & 0.05 & 0.07 & 0.03 & 0.05 & 0.24 & 0.010857 & 0.007311 \\
\hline I & 0.025 & 0.09 & 0.19 & 0.00 & 0.27 & 0.05 & 0.07 & 0.03 & 0.05 & 0.24 & 0.010976 & 0.007411 \\
II & 0.025 & 0.10 & 0.24 & 0.00 & 0.24 & 0.04 & 0.06 & 0.03 & 0.05 & 0.24 & 0.010962 & 0.007349 \\
III & 0.025 & 0.10 & 0.23 & 0.00 & 0.24 & 0.04 & 0.06 & 0.03 & 0.06 & 0.24 & 0.010963 & 0.007354 \\
IV & 0.025 & 0.10 & 0.23 & 0.00 & 0.24 & 0.04 & 0.06 & 0.03 & 0.05 & 0.24 & 0.010943 & 0.007352 \\
\hline
\end{tabular}


Table 6 confirms the fact that for different expected returns $E_{*}$ the nonparametric method based on median estimation lead to low risk, in comparison to the classical and Mean-DSR models. It is important to note that in terms of assets allocation there is no important difference for the parametric and nonparametric DSR methods.

In order to clarify whether the portfolios have been successfully optimised the results were compared with CAC 40 and Bovespa Indexes. For each method, 334 french portfolio returns are compared to $334 \mathrm{CAC} 40$ daily values. Figure 3 shows the daily difference between optimal portfolio and CAC 40 returns. Blue color indicates that the portfolio performs better. The comparison is made for different expected return level $E_{*}$. Figure 3 exhibits the results concerning the four methods for $E_{*}=0.03 \%$. Similarly, the daily differences between optimal Brazilian portfolio and BOVESPA Index returns for 2013's sample and for an expected return $E_{*}=0.03 \%$, are plotted in Figure 4 .
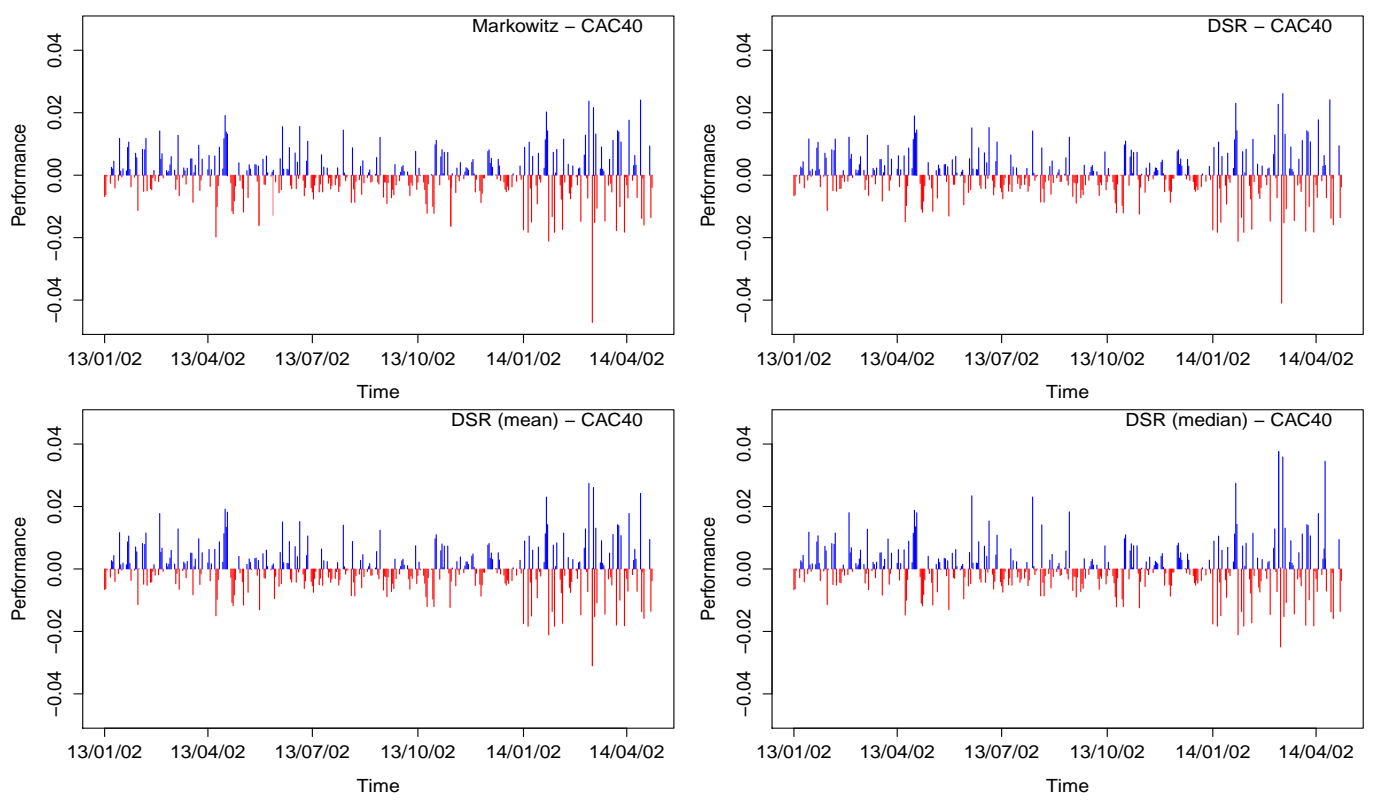

Figure 3: Portfolio returns against CAC 40 Index
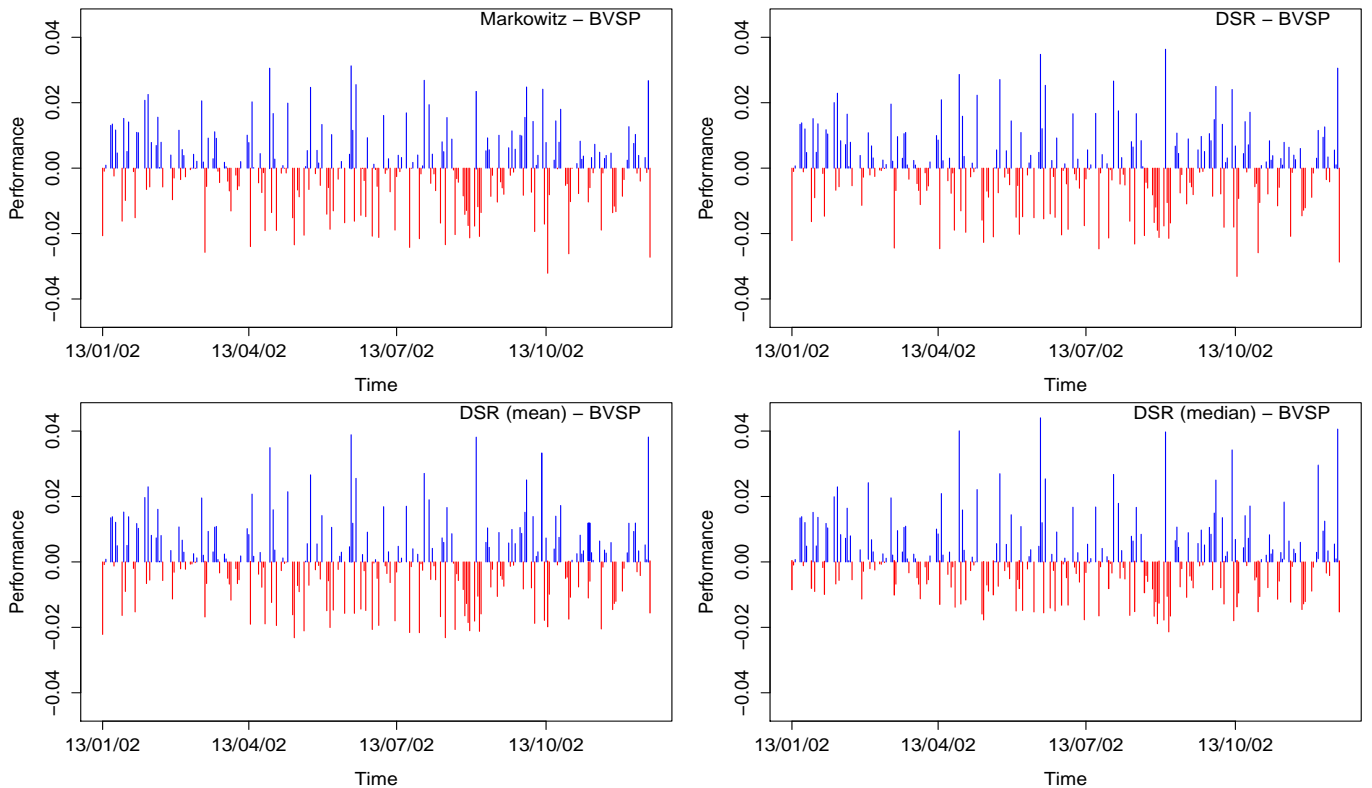

Figure 4: Portfolio returns against BOVESPA Index 
In the French market, it appears that the nonparametric methods are more efficient, in term of returns, than classical Markowitz and DSR models. Furthermore, the method based on kernel median estimation is more appealing than the one based on kernel mean estimation (especially at the end of studied period). The same conclusion is reached for different choices of $E_{*}$.

In the brazilian case, the portfolio returns obtained by nonparametric methods are better than BOVESPA Index. More precisely, the method using the smoothing median performs better.

These results will be confirmed later by using Sharpe and Sortino Ratios ( See 4.2.3).

\subsubsection{Optimization with short selling}

Combining long and short positions become increasingly popular among institutions such as hedge funds and individual investors, as traditional strategies struggled in a bear market, highlighting the need for investors to consider expanding their portfolios into innovative financial solutions and take profit from market evolution.

Long-short strategy consists of buying an undervalued stock and shorting or selling an overvalued stock. the short selling involves borrowing a stock from a broker and then selling it in the market. The stock is bought back and returned to the broker at a later date, this is called covering the short. If the stock drops, the short seller buys it back at a lower price and then he makes money.

Taking short positions provides a way to speculate if you think a market's value is going to decline. This allows you to add value to your portfolio even in a bear market. It is also a tool to hedge a portfolio.

In this section, short selling constraints $\left(\omega_{i} \in \mathbb{R}\right.$, for $\left.i=1, \ldots, 9\right)$ will be incorporated to resolve the different optimization problems (Classical Mean-Variance Markowitz, Classical DSR, nonparametric Mean DSR and nonparametric Median DSR Methods).

Once more two stock markets will be investigated: The French and Brazilian Stock Markets.

As the previous section, data of the French and Brazilian markets is divided into two parts: the first one for determining optimal portfolios and the second one for testing the efficiency of the methods. The last sample will be used to assess performance of the optimal portfolio returns against the CAC 40 and BOVESPA Indexes.

After imposing short Selling constraint, the four methods (classical Mean-Variance, classical DSR, DSR with mean smoothing, and DSR with median smoothing) are applied on the data in order to get optimal portfolios, and plot the correspondent efficient frontiers.

For the French case (Figure 5), it is apparent from the graphical comparison that classical and nonparametric DSR methods push the efficient frontiers outward to the left of the classical Mean-Variance curve to produce efficient portfolios that are stochastically dominant. The nonparametric efficient frontier based on median estimation stochastically dominates the other curves for any given expected return $E_{*}$.

In the Brazilian case (Figure 6), efficient frontiers in DSR framework surpass the classical Mean-Variance one. In fact, for any given expected return, the DSR efficient frontiers dominates stochastically the Mean-Variance curve. Although, the classical DSR seems performing slightly better than the nonparametric Mean DSR model, the nonparametric Median model still the most efficient one.

Table 7)(French market) and Table 8 (Brazilian market) confirm the obtained results, and give the optimal weights for different expected returns. 


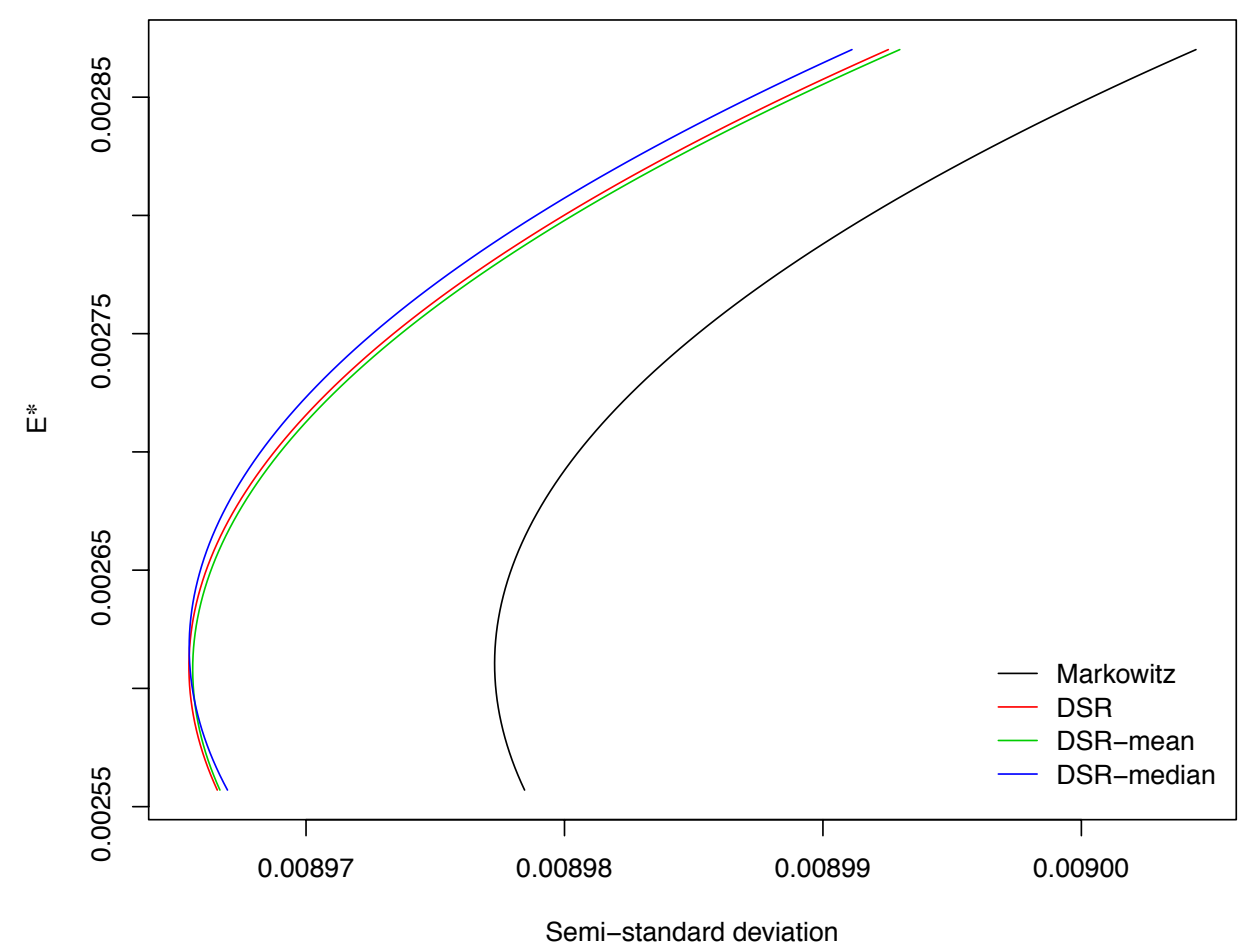

Figure 5: French Portfolio Efficient Frontiers $\left(\omega_{i} \in \mathbb{R}\right)$

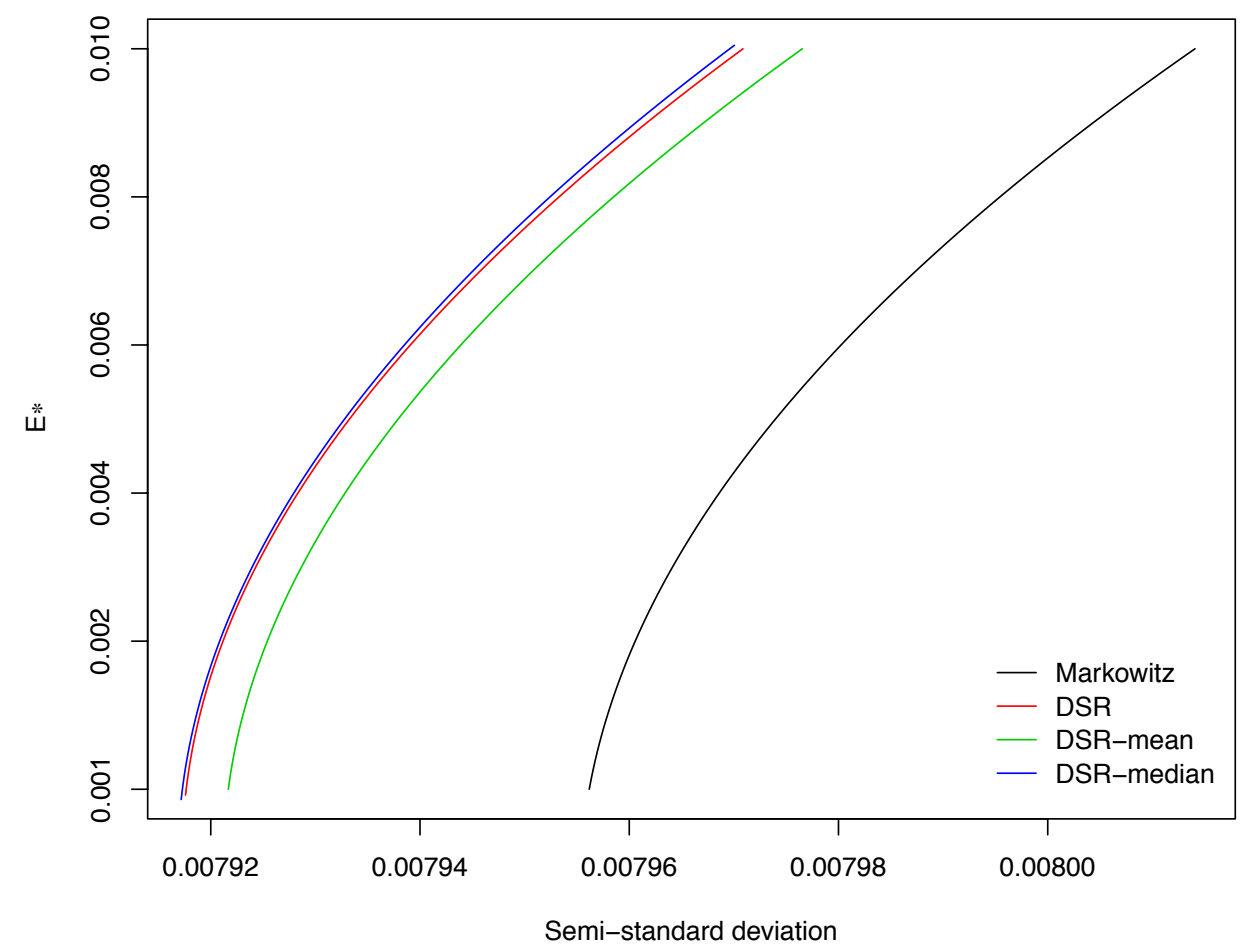

Figure 6: Brazilian Efficient Portflio Frontiers $\left(\omega_{i} \in \mathbb{R}\right)$ 
Table 7: France: Optimal portfolios with short selling

\begin{tabular}{lcccccccccccc}
\hline Model & $E_{*}$ & Accor & Airb & Alcat & BNP & Carr & Dano & Oréa & S.Gle & Total & S.D. & Semi-S.D. \\
\hline I & 0.025 & 0.10 & 0.04 & -0.05 & -0.02 & 0.07 & 0.41 & 0.22 & -0.05 & 0.26 & 0.013222 & 0.009196 \\
II & 0.025 & 0.11 & 0.05 & -0.05 & 0.01 & 0.08 & 0.38 & 0.24 & -0.08 & 0.26 & 0.013220 & 0.009183 \\
III & 0.025 & 0.11 & 0.05 & -0.05 & 0.01 & 0.08 & 0.38 & 0.24 & -0.09 & 0.27 & 0.013222 & 0.009183 \\
IV & 0.025 & 0.11 & 0.05 & -0.05 & 0.01 & 0.08 & 0.38 & 0.24 & -0.09 & 0.27 & 0.013200 & 0.009183 \\
\hline I & 0.030 & 0.11 & 0.07 & -0.07 & 0.00 & 0.02 & 0.43 & 0.24 & -0.05 & 0.25 & 0.013318 & 0.009231 \\
II & 0.030 & 0.11 & 0.07 & -0.08 & 0.04 & 0.03 & 0.41 & 0.25 & -0.08 & 0.25 & 0.013316 & 0.009219 \\
III & 0.030 & 0.11 & 0.07 & -0.07 & 0.04 & 0.03 & 0.40 & 0.25 & -0.08 & 0.26 & 0.013310 & 0.009219 \\
IV & 0.030 & 0.11 & 0.07 & -0.07 & 0.04 & 0.03 & 0.40 & 0.25 & -0.08 & 0.25 & 0.013296 & 0.009216 \\
\hline I & 0.035 & 0.11 & 0.09 & -0.10 & 0.02 & -0.03 & 0.45 & 0.26 & -0.04 & 0.24 & 0.013528 & 0.009346 \\
II & 0.035 & 0.12 & 0.09 & -0.10 & 0.06 & -0.02 & 0.43 & 0.27 & -0.08 & 0.24 & 0.013518 & 0.009333 \\
III & 0.035 & 0.12 & 0.09 & -0.10 & 0.06 & -0.03 & 0.42 & 0.27 & -0.08 & 0.24 & 0.013531 & 0.009334 \\
IV & 0.035 & 0.12 & 0.09 & -0.10 & 0.06 & -0.02 & 0.43 & 0.27 & -0.08 & 0.24 & 0.013508 & 0.009328 \\
\hline I & 0.040 & 0.12 & 0.11 & -0.13 & 0.04 & -0.09 & 0.47 & 0.28 & -0.04 & 0.23 & 0.013854 & 0.009538 \\
II & 0.040 & 0.12 & 0.11 & -0.13 & 0.09 & -0.08 & 0.45 & 0.28 & -0.07 & 0.23 & 0.013837 & 0.009524 \\
III & 0.040 & 0.12 & 0.11 & -0.12 & 0.09 & -0.08 & 0.44 & 0.28 & -0.07 & 0.23 & 0.013850 & 0.009526 \\
IV & 0.040 & 0.12 & 0.11 & -0.12 & 0.09 & -0.08 & 0.45 & 0.28 & -0.07 & 0.23 & 0.013829 & 0.009517 \\
\hline I & 0.045 & 0.13 & 0.13 & -0.15 & 0.06 & -0.14 & 0.49 & 0.30 & -0.03 & 0.22 & 0.014280 & 0.009804 \\
II & 0.045 & 0.13 & 0.13 & -0.15 & 0.11 & -0.13 & 0.47 & 0.30 & -0.07 & 0.21 & 0.014275 & 0.009789 \\
III & 0.045 & 0.13 & 0.13 & -0.15 & 0.11 & -0.14 & 0.46 & 0.30 & -0.07 & 0.21 & 0.014259 & 0.009792 \\
IV & 0.045 & 0.13 & 0.13 & -0.15 & 0.11 & -0.13 & 0.47 & 0.30 & -0.06 & 0.21 & 0.014252 & 0.009781 \\
\hline
\end{tabular}

For any expected return level, investors should take short positions on the Alcatel and Société Générale assets to construct the optimal portfolio. The Carrefour asset should be shorted for a high level of expected return. This result was expected because during the studied period, the financial markets, fearing the collapse of the Euro zone associated with the European sovereign debt crisis, were severely shaken. European and French bank shares, especially the Société Générale Paris share recorded substantial falls. In fact, the Bearish pressure, influenced by speculation but also by investor suspicion, continued to affect Société Générale's share price through to 2011 and 2012, the share losing 57.22\% of its value. During the studied period, Alcatel-Lucent has been at risk since 2006, because it missed key technological shifts. They unveiled plans to slash jobs worldwide, in order to stem years of losses, cut operating costs and consolidate resources around fewer sites as after losing 700-800 million ( $\$ 1.08$ billions) per year since its merger with U.S. firm Lucent Technologies in 2006. As to Carrefour, Europe's biggest retailer, they incured a loss of $2.6 \%$ in 2012, depressed by the falling demand in recession-hit Spain and Italy. The proportions invested on Total and L'Oréal are very important compared to the other assets contributions as they represent about $50 \%$ of the optimal portfolio. It is noted that, in the DSR framework, there is no huge difference in the proportions invested on the different assets. 
Table 8: Brazil: Optimal portfolios with short selling

\begin{tabular}{lcccccccccccc}
\hline Model & $E_{*}$ & B.Am. & B.Es. & P.Ga. & Cos & Inf & Idei & S.En & G.Int. & Oi.SA & S.D. & Semi-S.D \\
\hline I & 0.01 & 0.09 & 0.19 & 0.00 & 0.22 & 0.09 & 0.10 & 0.04 & 0.03 & 0.24 & 0.010747 & 0.007355 \\
II & 0.01 & 0.09 & 0.23 & 0.00 & 0.19 & 0.08 & 0.09 & 0.03 & 0.04 & 0.24 & 0.010743 & 0.007303 \\
III & 0.01 & 0.09 & 0.23 & 0.00 & 0.20 & 0.08 & 0.09 & 0.03 & 0.04 & 0.24 & 0.010726 & 0.007305 \\
IV & 0.01 & 0.09 & 0.23 & 0.00 & 0.20 & 0.08 & 0.09 & 0.03 & 0.04 & 0.24 & 0.010670 & 0.007302 \\
\hline I & 0.015 & 0.09 & 0.19 & 0.00 & 0.23 & 0.08 & 0.09 & 0.04 & 0.04 & 0.24 & 0.010786 & 0.007346 \\
II & 0.015 & 0.10 & 0.24 & -0.01 & 0.21 & 0.07 & 0.09 & 0.03 & 0.04 & 0.24 & 0.010782 & 0.007291 \\
III & 0.015 & 0.10 & 0.23 & -0.01 & 0.21 & 0.07 & 0.08 & 0.03 & 0.05 & 0.24 & 0.010764 & 0.007294 \\
IV & 0.015 & 0.10 & 0.23 & -0.01 & 0.21 & 0.07 & 0.08 & 0.03 & 0.04 & 0.24 & 0.010706 & 0.007291 \\
\hline I & 0.02 & 0.09 & 0.19 & -0.01 & 0.25 & 0.07 & 0.08 & 0.04 & 0.04 & 0.24 & 0.010856 & 0.007360 \\
II & 0.02 & 0.10 & 0.24 & -0.02 & 0.22 & 0.06 & 0.08 & 0.04 & 0.05 & 0.24 & 0.010852 & 0.007302 \\
III & 0.02 & 0.10 & 0.23 & -0.01 & 0.23 & 0.06 & 0.07 & 0.03 & 0.05 & 0.24 & 0.010833 & 0.007306 \\
IV & 0.02 & 0.10 & 0.23 & -0.02 & 0.22 & 0.06 & 0.08 & 0.04 & 0.05 & 0.24 & 0.010771 & 0.007303 \\
\hline I & 0.025 & 0.10 & 0.19 & -0.02 & 0.27 & 0.06 & 0.07 & 0.04 & 0.05 & 0.24 & 0.010957 & 0.007397 \\
II & 0.025 & 0.10 & 0.24 & -0.02 & 0.24 & 0.05 & 0.07 & 0.04 & 0.06 & 0.24 & 0.010934 & 0.007336 \\
III & 0.025 & 0.10 & 0.23 & -0.02 & 0.24 & 0.05 & 0.06 & 0.04 & 0.06 & 0.24 & 0.010953 & 0.007341 \\
IV & 0.025 & 0.10 & 0.23 & -0.02 & 0.24 & 0.05 & 0.07 & 0.04 & 0.06 & 0.24 & 0.010866 & 0.007338 \\
\hline
\end{tabular}

The results of Table 8 show that for different expected returns, there is no huge difference in assets allocation, specially in the DSR framework. It is found that the contribution of B.Esta, Cosan, and Oi.SA assets to optimal portfolio constitution is very important, so it represents about $67 \%$ of the long positions. It is important to note that investors, for high expected returns, should short the Pet.e.Gas asset. For any expected return level, the nonparametric Method based on Median Estimation present lower risk than the other models.

Following the same principle as previously (optimization without short selling), the difference between optimal portfolio and the CAC 40 Index returns of the four proposed models, are made for different expected return level $E_{*}$. Figure 7 exhibits this difference concerning the French market for the expected return $E_{*}=0.03$.
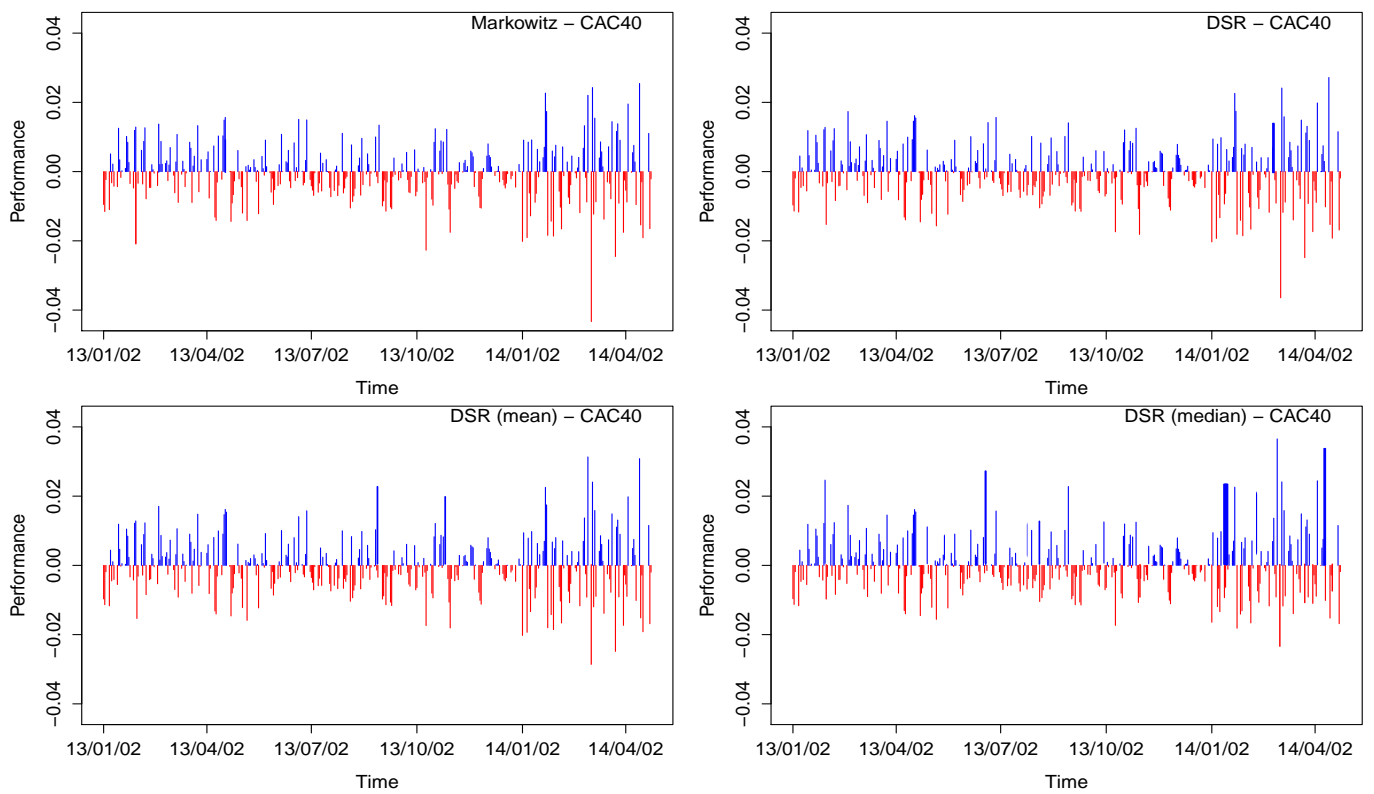

Figure 7: Portfolio returns against CAC 40 Index 
Similarly, the daily differences between optimal Brazilian portfolio and BOVESPA Index returns for 2013's sample and for the expected return $E_{*}=0.03 \%$, are plotted in Figure 8 .
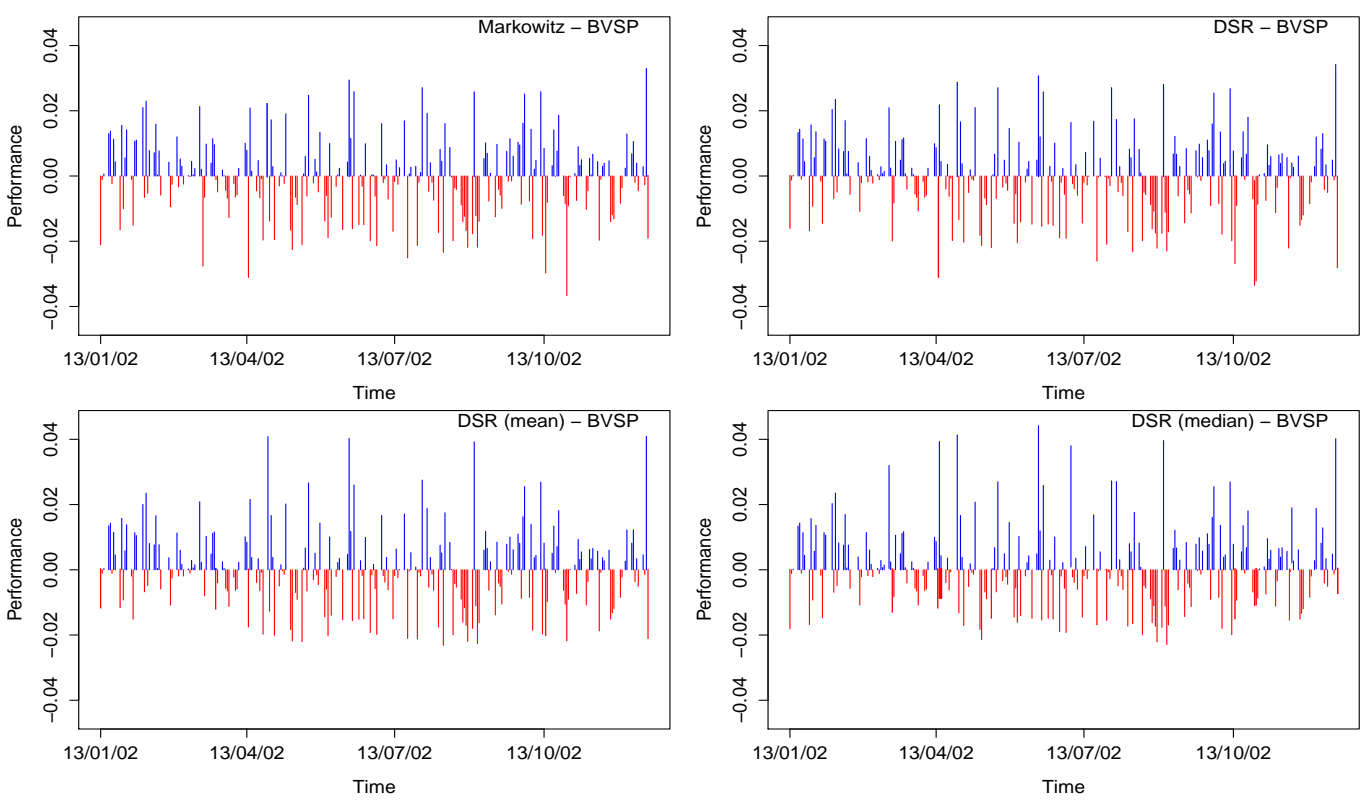

Figure 8: Portfolio returns against BOVESPA Index

For the French market (Figure 7), in the classical Mean-Variance framework, the portfolio returns are slightly lower than the CAC 40 returns. However in the parametric and nonparametric DSR framework, returns of the optimal portfolio are close and outperform the CAC 40 values specially at the end of the studied period. It is interesting to note that returns of optimal potfolio obtained by the Smoothing Median method are more efficient than the CAC 40 Index (returns).

In the Brazilian case, Figure 8 shows that, contrary to the classical Mean-Variance model, the classical DSR and nonparametric DSR based on mean estimation are slightly close to BOVESPA Index. Generally, nonparametric Median DSR performs better than BOVESPA Index.

Same conclusions are reached for different choices of $E_{*}$.

\subsubsection{Performance Analysis}

So as to determine the performance of the proposed (parametric and nonparametric) models, adopting different strategies (only long or long-short strategies), explicit measures of performance are investigated in this section. Indeed, two performance ratios : the Sharpe (ShR) and Sortino (SoR) ratios will be exhibited and analysed.

- Sharpe Ratio (Sharpe (1966))

The Sharpe ratio is the average return earned in excess of the risk-free rate per unit of volatility or total risk. Subtracting the risk-free rate from the mean return, the performance associated with risk-taking activities can be isolated. The Sharpe ratio is given by this formula

$$
S h R=\frac{E\left(R_{p}\right)-R_{f}}{\sigma\left(R_{p}\right)}
$$

where $E\left(R_{p}\right)$ denotes the expected return of the portfolio, $R_{f}$ the return on the risk-free asset and $\sigma\left(R_{p}\right)$ the standard deviation of the portfolio returns. 
This ratio is a relevant performance measure when returns are normally distributed. Abnormalities like kurtosis, fatter tails and higher peaks, or skewness on the distribution can be a problematic for the ratio, as standard deviation doesn't have the same effectiveness when these problems exist. That is why it is interesting to calculate the Sortino ratio.

- Sortino Ratio (Sortino (1994)):

Sortino Ratio is a transformation of the Sharpe ratio that takes into consideration only the downside deviations. The use of this ratio is recommended to analyse highly volatile portfolios or when when the distributions are asymmetric. A portfolio is considered as efficient one when it has high Sortino Ratio. The Sortino ratio is given by this formula:

$$
S o R=\frac{E\left(R_{p}\right)-\tau}{D S R}
$$

where $E\left(R_{p}\right)$ is the asset or portfolio average realized return, $\tau$ the target or required rate of return for the investment strategy under consideration (originally called the minimum acceptable return) and $D S R$ the target semi-deviation (the square root of target semi-variance).

Here $\tau=R_{f}$ is taken as the risk free rate of return given by the mean of government bonds returns.

Table 9 sums up the levels of Sharpe and Sortino Ratios for all the methods, for the French stock market:

Table 9: Performance Sharpe and Sortino Ratios for French Stock Market

\begin{tabular}{|c|c|c|c|c|c|c|c|c|}
\hline \multirow{2}{*}{$E_{*}$} & \multicolumn{4}{|c|}{ Sharpe ratio } & \multicolumn{4}{|c|}{ Sortino ratio } \\
\hline & $\mathrm{M}-\mathrm{V}$ & DSR & DSR-M & DSR-MD & $\mathrm{M}-\mathrm{V}$ & DSR & DSR-M & DSR-MD \\
\hline & \multicolumn{8}{|c|}{ Positive weights only } \\
\hline 0.025 & 0.020 & 0.021 & 0.021 & 0.022 & 0.030 & 0.031 & 0.031 & 0.032 \\
\hline 0.030 & 0.003 & 0.003 & 0.003 & 0.004 & 0.005 & 0.005 & 0.005 & 0.006 \\
\hline 0.035 & 0.013 & 0.014 & 0.014 & 0.015 & 0.019 & 0.019 & 0.019 & 0.020 \\
\hline 0.040 & -0.029 & -0.029 & -0.029 & -0.026 & -0.041 & -0.041 & -0.041 & -0.040 \\
\hline & \multicolumn{8}{|c|}{ Allowing negative weights } \\
\hline 0.025 & 0.030 & 0.040 & 0.040 & 0.042 & 0.058 & 0.059 & 0.059 & 0.061 \\
\hline 0.030 & 0.031 & 0.042 & 0.042 & 0.044 & 0.063 & 0.064 & 0.064 & 0.065 \\
\hline 0.035 & 0.060 & 0.069 & 0.069 & 0.072 & 0.104 & 0.105 & 0.105 & 0.108 \\
\hline 0.040 & 0.090 & 0.092 & 0.093 & 0.095 & 0.145 & 0.146 & 0.146 & 0.150 \\
\hline
\end{tabular}

These ratios show that nonparametric methods, specially that using DSR with Median smoothing have higher Sharpe and Sortino ratios which means that they are more efficient than the classical ones. It is interesting to note that the four methods with Short-Selling constraint, have higher Sharpe and Sortino ratios, which means that long-short strategy are more efficient then the long only strategy.

Similarly, the performance Sharpe and Sortino ratios for Brazilian Stock Market are exhibited in Table 10 
Table 10: Performance Sharpe and Sortino Ratios for Brazilian Stock Market

\begin{tabular}{|c|c|c|c|c|c|c|c|c|}
\hline \multirow{2}{*}{$E_{*}$} & \multicolumn{4}{|c|}{ Sharpe ratio } & \multicolumn{4}{|c|}{ Sortino ration } \\
\hline & $M-V$ & DSR & DSR-M & DSR-MD & $\mathrm{M}-\mathrm{V}$ & DSR & DSR-M & DSR-MD \\
\hline & \multicolumn{8}{|c|}{ without short selling } \\
\hline 0.005 & -0.077 & -0.074 & -0.074 & -0.073 & -0.100 & -0.097 & -0.097 & -0.097 \\
\hline 0.010 & -0.074 & -0.073 & -0.073 & -0.071 & -0.097 & -0.096 & -0.096 & -0.094 \\
\hline 0.015 & -0.073 & -0.073 & -0.073 & -0.071 & -0.096 & -0.095 & -0.096 & -0.094 \\
\hline 0.020 & -0.073 & -0.072 & -0.072 & -0.071 & -0.096 & -0.095 & -0.095 & -0.095 \\
\hline 0.025 & -0.074 & -0.072 & -0.072 & -0.072 & -0.096 & -0.095 & -0.095 & -0.094 \\
\hline \multicolumn{9}{|c|}{ with short selling } \\
\hline 0.005 & -0.076 & -0.073 & -0.073 & -0.070 & -0.099 & -0.096 & -0.096 & -0.093 \\
\hline 0.010 & -0.074 & -0.071 & -0.072 & -0.071 & -0.097 & -0.093 & -0.094 & -0.093 \\
\hline 0.015 & -0.071 & -0.068 & -0.069 & -0.068 & -0.094 & -0.089 & -0.090 & -0.089 \\
\hline 0.020 & -0.068 & -0.065 & -0.066 & -0.065 & -0.090 & -0.086 & -0.086 & -0.085 \\
\hline 0.025 & -0.065 & -0.062 & -0.062 & -0.061 & -0.086 & -0.081 & -0.082 & -0.080 \\
\hline
\end{tabular}

Although the Sharp and Sortino ratios are negative for any given expected return, they still a tool to compare the performance of all the methods. Indeed, the nonparametric methods, and more precisely the one based on median estimation, still slightly better than the classical models. Contrary to our expectations, in terms of performance, Longshort strategy does not perform significantly the only long strategy witch means that the Brazilian market in that period is bullish, and shorting assets in this context are not an appropriate way to make profit. Graphic 99) shows that the Brazilian Stock Market follows slightly upward trend during the testing period. However, the sample data used in the optimization step presents a flat trend.
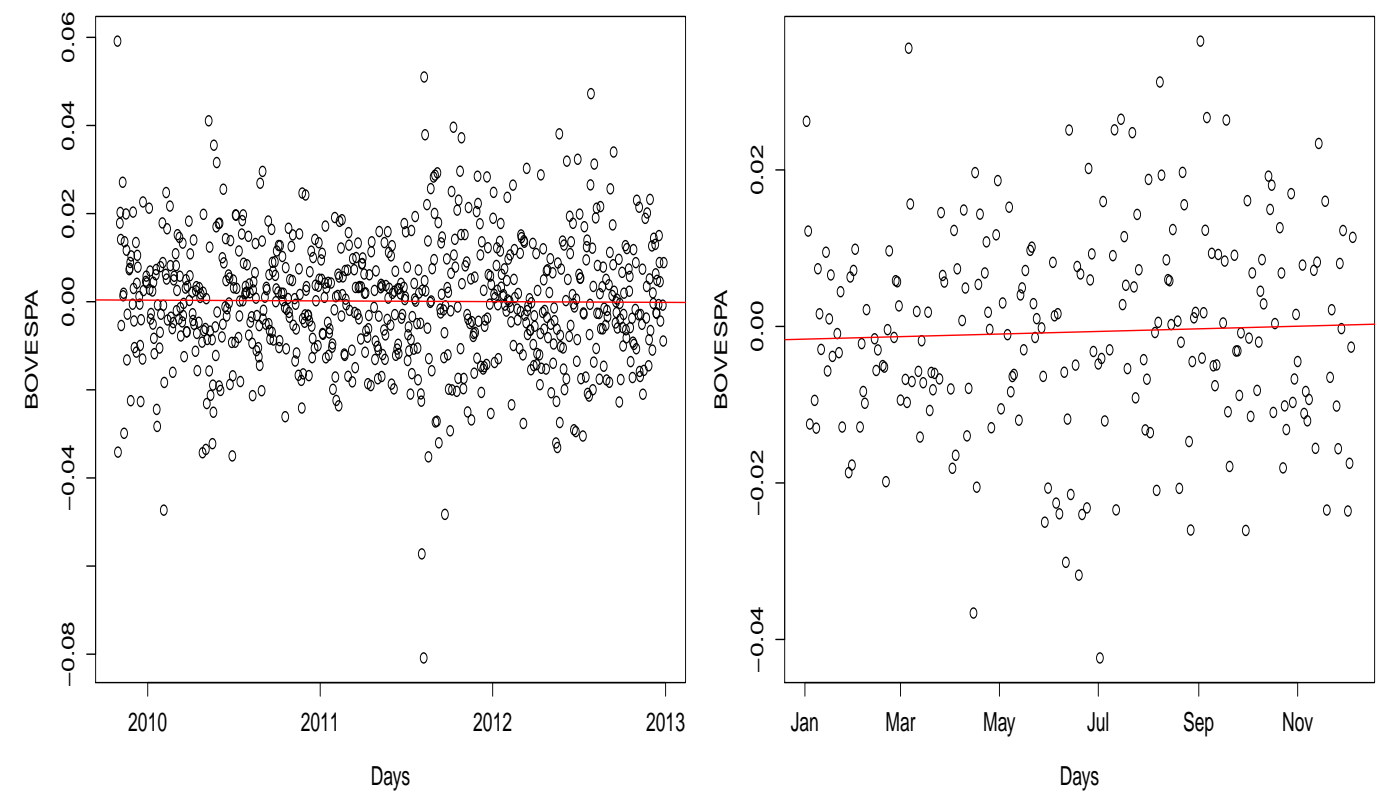

Figure 9: Brazilian Market Trend

\section{Conclusion}

The classical Mean-Variance model of Markowitz is one of the most popular used models for determining optimal portfolio weights. However, it was widely criticised because its strict assumptions related to the normality or to the 
symmetry of returns distribution. Many proposals of new risk measures are developed in the literature in order to consider downside movements because investors are more concerned and averse about the DownSide risks.

Our contribution consists on the introduction of nonparametric approach in the estimation of the DownSide Risk. It leads to get smoother efficient frontiers. In addition, adequate iterative algorithms were developed to resolve the optimization problems. The proposed method based on median estimation is precise and very important in term of performance thanks to the robustness of the median. Also, in term of computation time, the proposed algorithms outperform the algorithm proposed by Ben Salah et al (2015).

Nonparametric methods, specially the Mean-DSR with median smoothing, provides lower portfolio risk compared to the classical Mean-Variance and Mean-Semivariance models.

The proposed DownSide Risk versions proposed in this paper were applied to determine the optimal weights in two different stock markets: Brazilian and French stock markets. The results show that the strategies of investment, in terms of asset allocation depend on the methods, and the risk measure used to optimize the portfolio. Asset allocation depends also on the kind of constraints used in the optimisation program like allowing short-selling or not.

It was pointed out that, the nonparametric methods for the French Stock Market, present lower risk comparing to the classical Mean-Variance and Classical Mean-DSR models. In term of performance, the nonparametric methods, specially the one based on median estimation, outperforms the CAC 40 Index in the two investigated strategies (long only strategy, long-short strategy). It is also interesting to note that nonparametric Methods for the French stock market have higher Sharpe and Sortino ratios.

The fact that the Sortino ratios are also much higher for long-short portfolios shows that, by allowing short selling, the downside risk (as measured by the target Semi-Variance) can be substantially reduced.

Our numerical experiments, for the Brazilian stock market, confirm the efficiency of the nonparametric Methods specially the one based on Median Estimation.

Indeed, the nonparametric Methods present lower risk than the classical one. In addition, the comparison to BOVESPA Index shows that nonparametric methods outperforms the classical ones.

Despite the negative values of Sharpe and Sortino ratios, it was clear that Median-DSR methods is more efficient than the classical DSR and Mean-DSR models.

It is believed that Long-Short portfolios are riskier than the long only portfolios (due to potentially unlimited losses on the short positions), consequently the long short strategy have to be more efficient.

However, our findings show, for the Brazilian stock market, the Long only strategy was more efficient than the Long-Short strategy. In fact, the choice of strategies should be appropriate to the nature of the market, so shorting in bullish market is not a successful strategy.

Our empirical studies were limited to stock markets, it will be interesting to test our methods on other assets (Bonds, Options, ....).

It is also interesting to focus on another DSR measures concerned with the left tails of distributions such as VaR and $\mathrm{CVaR}$.

\section{References}

Ang, J. (1975). A note on the ESL Portfolio Selection Model. Journal of Financial and Quantitative Analysis, 10, 849-857.

Arlot, S., Celisse, A. (2010). A survey of cross-validation procedures for model selection. Statistics Surveys, Vol. 4 (2010) 40-79.

Athayde, G. (2001). Building a Mean-Downside Risk Portfolio Frontier, Developments in Forecast Combination and Portfolio Choice, John Wiley and Sons.

Athayde, G. (2003). The mean-downside risk portfolio frontier : a non-parametric approach Published in Advances in portfolio construction and implementation.

Ben Salah, H., Chaouch, M., Gannoun, A., de Peretti, C., Trabelsi, A. (2015). Mean and Median-based nonparametric Estimation of Returns in Mean-DownSide Risk Portfolio Frontier. Submitted.

Berlinet, A., Gannoun, A., Matzner-Løber, E. (2001). Asymptotic of convergent estimates of conditional quantiles. Statistics, 35, 139-169.

Eftekhari, B., Satchel, S. (1996). Non-Normality of Returns in Emerging Markets. Research in International Business and Finance, Supplement 1, 267-277. 
Estrada, J. (2004). Mean-Semivariance Behavior : An Alternative Behavioural Model. Journal of Emerging Market Finance, 3, 231-248.

Estrada, J. (2008). Semivariance Optimization : A Heuristic Approach. Journal of Applied Finance, 57-72.

Gannoun, A., Saracco, J., Yu, K.(2003). nonparametric time series prediction by conditional median and quantiles. Journal of statistical Planning and inference, 117, 207-223.

Harlow, V. (1991). Asset allocation in a downside risk framework. Financial Analyst Journal, 47, 28-40.

Hogan, W., Warren, J. (1974). Computation of the efficient Boundary in the ES Portfolio selection. Journal of Financial and Quantitative Analysis, 9, 1-11.

Koenker, R. (2005). Quantile Regression, Econometric Society Monographs, Cambridge University Press.

Markowitz, M., Peter,T., Ganlin, X., Yuji,Y. (1993). Computation of mean-semivariance Efficient sets by the Critical Line Algorithm. Annals of Operationss Research, 45, 307-317.

Mamoghli, C., Daboussi, S. (2010). Optimisation de portefeuille downside risk. J. Emerg Market Finance, 9, no. 2, 95-130.

Markowitz, H. (1952). Portfolio Selection, Journal of Finance, 7, 77-91.

Markowitz, H.M. (1959). Portfolio Selection : Efficient Diversification of Investments. New York : John Wiley \& Sons.

Markowitz, H.M. (1991). Portfolio Selection : Efficient Diversification of Investments. Basil Blakwell.

Markowitz, H. M., Todd, P., Xu, G., Yamane, Y. (1993). Computation of mean-semivariance efficient sets by the critical line algorithm. Annals of Operations Research, 45, 307-317.

Pagan, A.R., Ullah, A. (1999). nonparametric Econometrics, Cambridge University Press.

Sharpe, W. (1966). Mutual Fund Performance. Journal of Business , 39, 119-138.

Silverman, B.W. (1986). Density Estimation for Statistics and Data Analysis. Chapman and Hall, New York.

Sortino, F. , L. Price.(1994). Performance measurement in a Downside risk framework. Journal of Investing, 3, 59-65. 


\section{Abbreviations}

Table 11: Companies abbreviations

\begin{tabular}{|l|l|l|l|}
\hline \multicolumn{2}{|c|}{ France } & \multicolumn{2}{c|}{ Brazil } \\
\hline Companies & Abb. & Companies & $A b b$. \\
\hline Accor SA & Accor & Banco PanAmericano SA & B.Amer \\
Airbus Group NV & Airb & Banco do Estado de Sergipe SA & B.Esta \\
Alcatel Lucent SA & Alcat & Ogx Petroleo e Gas Participacoes SA & Pet.e.Gas \\
BNP Paribas SA & BNP & Cosan Ltd & Cosan \\
Carrefour SA & Carr & Positivo Informatica SA & Informati \\
Danone SA & Dano & Ideiasnet SA & Ideasnet \\
L'Oreal SA & Oréa & Mills Estruturas e Servicos de Engenharia SA & S.Enge \\
Sociéte Générale & S.Gle & Gol Linhas Aereas Inteligentes SA & G.Intel \\
Total SA & Total & Oi SA & Oi.SA \\
\hline
\end{tabular}

